\title{
Instability of dislocation fluxes in a single slip: Deterministic and stochastic models of dislocation patterning
}

\author{
Ronghai Wu, ${ }^{1,2}$ Daniel Tüzes, ${ }^{3}$ Péter Dusán Ispánovity, ${ }^{3}$ István Groma, ${ }^{3}$ Thomas Hochrainer, ${ }^{4}$ and Michael Zaiser ${ }^{2,5},{ }^{*}$ \\ ${ }^{1}$ School of Mechanics, Civil Engineering, and Architecture, Northwestern Polytechnical University, Xian 710129, People's Republic of China \\ ${ }^{2}$ Institute for Materials Simulation, Department of Materials Science, Friedrich-Alexander University Erlangen-Nürnberg (FAU), \\ Dr.-Mack-Straße 77, 90762 Fürth, Germany \\ ${ }^{3}$ Department of Materials Physics, ELTE, Eötvös Loránd University, H-1517 Budapest POB 32, Hungary \\ ${ }^{4}$ Institut für Festigkeitslehre, Technische Universität Graz, Kopernikusgasse 24/I, 8010 Graz, Austria \\ ${ }^{5}$ Department of Mechanics and Engineering, Southwest Jiaotong University, Chengdu, People's Republic of China
}

(Received 21 August 2017; revised manuscript received 18 December 2017; published 24 August 2018)

\begin{abstract}
We study a continuum model of dislocation transport in order to investigate the formation of heterogeneous dislocation patterns. We propose a physical mechanism that relates the formation of heterogeneous patterns with a well-defined wavelength to the stress-driven dynamics of dislocation densities that tries to minimize the internal energy while subject to dynamic constraints and a density-dependent, friction-like flow stress. This leads us to an interpretation that resolves the old "energetic vs dynamic" controversy regarding the physical origin of dislocation patterns and emphasizes the hydrodynamic nature of the instability that leads to dislocation patterning, which we identify as an instability of dislocation transport that is not dependent on processes such as dislocation multiplication or annihilation. We demonstrate the robustness of the developed patterning scenario by considering the simplest possible case (plane strain, single slip) in two model versions that consider the same driving stresses but implement the transport of dislocations that controls dislocation density evolution in two very different manners, namely (i) a hydrodynamic formulation that considers transport equations that are continuous in space and time, assuming that the dislocation velocity depends linearly on the local driving stress, and (ii) a stochastic cellular automaton implementation that assumes spatially and temporally discrete transport of discrete "packets" of dislocation density that move according to an extremal dynamics. Despite the differences, we find that the emergent patterns in both models are mutually consistent and in agreement with the prediction of a linear stability analysis of the continuum model. We also show how different types of initial conditions lead to different intermediate evolution scenarios that, however, do not affect the properties of the fully developed patterns.
\end{abstract}

DOI: 10.1103/PhysRevB.98.054110

\section{INTRODUCTION}

Ever since the first TEM observations of dislocations it has been known that the arrangement of dislocations in deformed crystals is practically never homogeneous: dislocations show an intrinsic propensity to form heterogeneous patterns. There is an equally long-standing discussion regarding the physical nature of these patterns that is matched by an amazing variety of approaches to their modeling, many of which are based on analogies with pattern formation in other physical systems. Thus, it has been argued that dislocation patterns form because the dislocations try to minimize elastic energy (see, e.g., Hansen and Kuhlmann-Wilsdorf [1]). Unfortunately this "energetic" approach to dislocation patterning has hardly ever been cast into a tangible mathematical framework by actually formulating and minimizing the energy functional in question. One notable exception is represented by the work of Holt [2], which is clearly crafted in analogy with contemporary models of spinodal decomposition patterns and relates the patterning of dislocation densities to the minimization of an associated internal energy functional, a process that in stark contrast

*michael.zaiser@fau.de to experiment is predicted to occur even in the absence of external stress. The "energetic" approach may be contrasted with the idea that dislocations in a deforming crystal constitute a driven system far from equilibrium where patterns may form as dissipative structures. This has led to the formulation of nonlinear sets of partial differential equations for dislocation densities by Walgraef and Aifantis [3] and Pontes et al. [4] that give rise to a variety of interesting patterns. Some of these resemble dislocation patterns observed by TEM, while other types of patterns predicted by the same equations, such as spiral waves, have never been observed [5]. In our opinion most of these models suffer from an overly phenomenological approach to modeling: They aim at reproducing patterns (actually, pictures of patterns) rather than deriving them from the known elastic and kinematic properties of dislocation systems. As a consequence, the fundamental controversy as to whether dislocation patterning is in essence an energetic or a dynamic phenomenon, which has been neatly summarized by Nabarro [6], remains unresolved.

Only in recent years have attempts been made to formulate dislocation density based models by means of averaging procedures that lead from the dynamics of discrete dislocations to the evolution of dislocation densities in a systematic manner. These methods have been used to formulate the kinematics of 
dislocations in 2D and lately in 3D [7-9] and also to systematically derive driving forces for the dynamics. To the latter end, two alternative approaches have been pursued: Driving forces for dislocation density evolution may be obtained by directly averaging the interaction forces of discrete dislocations [10-12]. Alternatively, one may formulate an energy functional governing the dynamics based on phenomenological considerations $[13,14]$ or from direct averaging of the elastic energy of the discrete dislocation system [15], and then obtain driving forces from variation of the energy functional in conjunction with thermodynamic consistency requirements [16]. Both approaches have been shown to yield mutually consistent results provided that, in the variational approach, a nontrivial mobility function is assumed which implements a friction stress [12].

Importantly, these statistical averaging approaches do not pursue the primary aim of "modeling patterning," i.e., of reproducing experimental images in a more or less faithful manner. Rather, such models aim at "modeling dislocations": their primary thrust is to provide a physically and mathematically consistent representation of the motion and interactions of dislocations in a continuum framework ("continuum dislocation dynamics," CDD), just as discrete dislocation dynamics simulations (DDD) aim at doing so in a discrete framework. Similarly to DDD simulations, where dislocation lines are quite generally found to entangle in a heterogeneous manner which is matched by the emergence of micro-heterogeneous deformation patterns (see, e.g., [17-19]), it has soon become clear that in CDD models the emergence of heterogeneous dislocation patterns turns out to be an almost inevitable feature of the collective dynamics. Simulations of CDD models in 3D demonstrate an intrinsic tendency towards dislocation patterning $[20,21]$ as they relate the emergence of patterning to the same dislocation interactions that govern strain hardening, in line with the "principle of similitude" [22]. This principle has been related to fundamental invariance properties of the equations that govern the properties of discrete dislocation systems, and indeed all physically based models of dislocation patterning published in recent years are consistent with these invariance properties [23].

While most recent models exploit advances in computational power [21] or in kinematic averaging methods [20] in order to address the important problem of dislocation patterning in 3D and under conditions of multiple slip, the present authors have pursued a more simplistic yet more fundamental goal, namely to elucidate the fundamental nature of the instability underlying dislocation patterning by investigating a most simple model, and then demonstrating that the basic mechanism of patterning in this model remains robust upon generalization. To this end we focus on a minimal system (2D, single slip) where an exact representation of the kinematics is possible and well-defined forms have been established both for the energy functional $[13,15]$ and also for the effective mobility law [12]. As a consequence, we can dispense to a large extent with phenomenological assumptions and obtain a complete understanding of the interplay of energy minimization, external driving, and friction in driving the emergence of dislocation patterns. In a previous work [12] we analyzed the linear stability of the ensuing equations under the assumption of a linear stress dependency of the collective velocity of dislocations. While this allowed us to reach important conclusions regarding the patterning mechanism, a linear stability analysis is of necessity insufficient to decide on the stability of the emergent patterns and the robustness of the patterning scenario. Furthermore, even if the velocity of single dislocations depends linearly on the local stress acting on the dislocation line, the same is not necessarily true for the collective velocity of interacting dislocations: Nontrivial collective velocity exponents resulting from a linear stress-velocity relationship on the single-dislocation level have been reported in simulations of 2D dislocation systems by Miguel et al. [24].

We therefore, in the present paper, combine linear stability analysis for a generic nonlinear stress-velocity law with a comprehensive study of nonlinear aspects of patterning including the influence of initial condition, pattern growth mode, and investigation of pattern stability as well as an investigation of the influences of model implementation (continuous vs discrete, deterministic vs stochastic) and velocity law. The paper is organized as follows: In Sec. II, we briefly present the continuum model formulated by Groma et al. [12], its generalization to a generic nonlinear stress-velocity relationship, and its implementation in a spatially and temporally continuous setting. Section III presents a spatially and temporally discrete stochastic model that considers the same spatial couplings and friction rules, but implements a completely different dynamics that corresponds to the limit of an infinite stress exponent of dislocation velocity. Results from both models are presented in Sec. IV and compared to results from linear stability analysis. A discussion concerning the nature and robustness of dislocation patterning is presented in the Conclusions.

\section{DETERMINISTIC CONTINUUM MODEL}

In the following we give a brief summary of the continuum model of dislocation transport developed by Groma et al. [10] and Groma et al. [12] (see also Valdenaire et al. [11]), where a detailed discussion of the derivation and statistical averaging methodology can be found. We consider a 2D system of straight parallel edge dislocations of both signs which can be envisaged as charged point particles moving in a perpendicularly intersecting plane (taken to be the $x y$ plane). Dislocations of Burgers vector modulus $b$ are assumed to move on a single slip system which constrains their motion to the slip direction which we identify with the $x$ direction.

\section{A. Transport equations}

The model equations have the structure of continuity equations. The stress-driven motion of a dislocation depends on its sign; we use the sign convention that, under a positive resolved shear stress, positive dislocations of density $\rho^{+}$move with velocity $v^{+}$in the $+x$ and negative dislocations of density $\rho^{-}$ move with velocity $v^{-}$in the $-x$ direction. These motions produce a shear strain $\gamma$ at rate

$$
\partial_{t} \gamma=b\left(\rho^{+} v^{+}+\rho^{-} v^{-}\right)
$$

Neglecting dislocation generation or annihilation, the transport equations have the simple structure

$$
\begin{aligned}
& \partial_{t} \rho^{+}(\boldsymbol{r}, t)=-\partial_{x}\left(\rho^{+} v^{+}\right), \\
& \partial_{t} \rho^{-}(\boldsymbol{r}, t)=\partial_{x}\left(\rho^{-} v^{-}\right),
\end{aligned}
$$


where the velocities are assumed to depend on local driving stresses in a generic nonlinear manner,

$$
\begin{aligned}
& v^{+}=M_{0} b \operatorname{sgn}\left(\mathcal{T}^{+}\right)\left(\left|\mathcal{T}^{+}\right|\right)^{m}, \\
& v^{-}=M_{0} b \operatorname{sgn}\left(\mathcal{T}^{-}\right)\left(\left|\mathcal{T}^{-}\right|\right)^{m} .
\end{aligned}
$$

In these equations, the $\mathcal{T}^{ \pm}$are effective shear stresses driving the motion of positive and negative dislocations and $M_{0}$ is a dislocation mobility coefficient (inverse drag coefficient). An alternative formulation of the transport equations can be given in terms of the total dislocation density $\rho=\rho^{+}+\rho^{-}$and the excess or geometrically necessary density $\kappa=\rho^{+}-\rho^{-}$as

$$
\begin{aligned}
& \partial_{t} \rho(\boldsymbol{r}, t)=-\partial_{x}\left(\rho^{+} v^{+}-\rho^{-} v^{-}\right)=-\partial_{x}\left(\rho v^{\mathrm{d}}+\kappa v^{\mathrm{m}}\right), \\
& \partial_{t} \kappa(\boldsymbol{r}, t)=-\partial_{x}\left(\rho^{+} v^{+}+\rho^{-} v^{-}\right)=-\partial_{x}\left(\rho v^{\mathrm{m}}+\kappa v^{\mathrm{d}}\right),
\end{aligned}
$$

where $v^{\mathrm{m}}=\left(v^{+}+v^{-}\right) / 2$ is the mean velocity of positive and negative dislocations and $v^{\mathrm{d}}=\left(v^{+}-v^{-}\right) / 2$ the velocity difference (see also Zhu et al. [25]).

\section{B. Evaluation of the effective driving stresses}

The effective driving stresses $\mathcal{T}\left(\tau_{\mathrm{d}}^{ \pm}, \tau_{\mathrm{f}}^{ \pm}\right)$result from the combination of sign-dependent local driving stresses $\tau_{\mathrm{d}}^{ \pm}$and friction-like stresses $\tau_{\mathrm{f}}^{ \pm}$as

$$
\mathcal{T}\left(\tau_{\mathrm{d}}^{ \pm}, \tau_{\mathrm{f}}^{ \pm}\right)= \begin{cases}\operatorname{sgn}\left(\tau_{\mathrm{d}}^{ \pm}\right)\left(\left|\tau_{\mathrm{d}}^{ \pm}\right|-\tau_{\mathrm{f}}^{ \pm}\right), & \left|\tau_{\mathrm{d}}^{ \pm}\right|>\tau_{\mathrm{f}}^{ \pm}, \\ 0, & \left|\tau_{\mathrm{d}}^{ \pm}\right| \leqslant \tau_{\mathrm{f}}^{ \pm} .\end{cases}
$$

The driving stresses are given by combinations of a spatially homogeneous shear stress $\tau_{\text {ext }}$ arising from remotely applied boundary tractions, which provides the external driving force for dislocation motion and plastic flow, and a set of stress contributions describing dislocation interactions,

$$
\tau_{\mathrm{d}}^{ \pm}=\tau_{\text {ext }}+\tau_{\text {int }}+\tau_{\text {back }} \pm \tau_{\text {diff }} .
$$

For 2D dislocation systems as studied here, the interaction stress contributions have been derived from a statistical analysis of dislocation correlations. This analysis was presented independently by Groma et al. [12] and Valdenaire et al. [11]. Here we only discuss the resulting expressions, considering the interaction stress contributions $\tau_{\text {int }}, \tau_{\text {back }}$, and $\tau_{\text {diff }}$ in turn:

(1) The "internal" shear stress $\tau_{\text {int }}$ arises from heterogeneity of the plastic eigenstrain $\boldsymbol{\varepsilon}_{\mathrm{pl}}=\gamma\left(\boldsymbol{e}_{x} \otimes \boldsymbol{e}_{y}+\boldsymbol{e}_{y} \otimes \boldsymbol{e}_{x}\right) / 2$. This stress can be calculated in various manners, e.g., by direct convolution of the dislocation shear stress field with the excess dislocation density $\kappa=\rho^{+}-\rho^{-}=-\partial_{x} \gamma / b$ as done by Groma et al. [10], by using an Airy stress function formalism [13], or numerically by solving the eigenstrain problem using finite elements. Here we use the method based on $\kappa$, setting

$$
\tau_{\text {int }}(\boldsymbol{r})=\int \kappa\left(\boldsymbol{r}^{\prime}\right) \tau_{x y}^{\mathrm{e}}\left(\boldsymbol{r}-\boldsymbol{r}^{\prime}\right) d^{2} r^{\prime},
$$

where $\tau_{x y}^{\mathrm{e}}$ is the shear stress field of a positive edge dislocation with the Fourier transform

$$
\tau_{x y}^{\mathrm{e}}(\boldsymbol{k})=-\frac{\mu}{\pi(1-v)} \frac{i k_{x} k_{y}^{2}}{k^{4}},
$$

where $\mu$ is the shear modulus of the material, $v$ is Poisson's ratio, and $k_{x}$ and $k_{y}$ are components of the Fourier wave vector with modulus $k$.
(2) The "back stress" $\tau_{\text {back }}$ counteracts accumulation of dislocations of the same sign. It is given by

$$
\tau_{\text {back }}(\boldsymbol{r})=-\mu b \frac{D}{\rho} \partial_{x} \kappa(\boldsymbol{r})=\mu \frac{D}{\rho} \partial_{x x} \gamma(\boldsymbol{r}),
$$

where $D$ is a nondimensional factor of the order of unity. We see that the back stress is proportional to the second gradient of the shear strain.

(3) Finally, the "diffusion stress" $\tau_{\text {diff }}$ is given by

$$
\tau_{\text {diff }}(\boldsymbol{r})=-\mu b \frac{A}{\rho} \partial_{x} \rho(\boldsymbol{r}),
$$

where $A$ is another nondimensional factor of the order of unity. The terminology "diffusion stress" is used because this stress, if inserted via Eqs. (6), (5), (3) into the transport equations Eq. (2), gives rise to a diffusion-like contribution to the evolution of the total dislocation density $\rho$.

Groma et al. [12] observed that the stress contributions $\tau_{\text {int }}, \tau_{\text {back }}$, and $\tau_{\text {diff }}$ represent kinematic hardening contributions. Indeed all these stress contributions can, via variational calculus, be derived from an energy functional of the dislocation system given by

$$
\begin{aligned}
E & =E_{\mathrm{el}}+E_{\mathrm{dis}}, \\
E_{\mathrm{el}} & =\iint \gamma(\boldsymbol{r}) \mathcal{G}\left(\boldsymbol{r}-\boldsymbol{r}^{\prime}\right) \gamma\left(\boldsymbol{r}^{\prime}\right) d^{2} r d^{2} r^{\prime}, \\
E_{\mathrm{dis}} & =\int \mu b^{2}\left(A \rho \ln (\rho)+\frac{D}{2} \frac{\kappa^{2}}{\rho}\right) d^{2} r .
\end{aligned}
$$

The first contribution to this functional represents the elastic energy associated with the average plastic eigenstrain $\gamma$, whereas the second term represents a correction that captures elastic energy contributions due to stress and strain heterogeneities on the scale of single dislocations, which cannot be represented in terms of the coarse-grained strain variable $\gamma$. For a formal derivation of these terms by averaging the elastic energy of the underlying discrete dislocation system, see Zaiser [15].

The "friction stresses" $\tau_{\mathrm{f}}^{ \pm}$in the effective stress expressions (5) are given by

$$
\tau_{\mathrm{f}}^{ \pm}=\alpha \mu b \sqrt{\rho}\left(1 \mp \frac{\kappa}{\rho}\right) .
$$

These stresses are of a different nature from the driving stresses: they represent friction-like, isotropic hardening contributions. While these stresses arise naturally from direct averaging of the dislocation interactions, they cannot be derived from an energy functional but need to be added "by hand" to an energy-based formalism where they enter in terms of a nontrivial, nonlinear mobility function with a mobility threshold [12]. The functional form of these stresses is that of Taylor stresses; in physical terms, they represent the mutual trapping of positive and negative dislocations into dipolar or multipolar configurations. Their dependency on $\kappa$ reflects the fact that the presence of an excess of dislocations of one sign implies reduced pinning of the majority and enhanced pinning of the minority population. In particular, for $\kappa=\rho$ or $\kappa=-\rho$ (only positive or only negative dislocations) the pinning stress is zero. 


\section{Physical interpretation}

Before we proceed to analyzing our equations, it is important to clarify the origin and physical nature of the different stress contributions and the meaning of the nondimensional constants $\alpha, D$, and $A$ which, in addition to material parameters, enter the model equations. To this end, it is important to emphasize that we formulate a continuum description on scales above the spacing of individual dislocations, such that in general multiple dislocations may be contained within a computational volume element. In fact, the mean dislocation spacing (MDS) may serve as a length scale that bounds the applicability of our equations from below. This distinguishes our equations from discrete-continuum transitions as proposed, e.g., by Lemarchand et al. [26]: There the plastic slip, and by extension the dislocation core, is coarse-grained over a volume less than the dislocation spacing; one is in these works, in essence, dealing with a continuous formulation of discrete dislocation dynamics where elastic dislocation interactions are completely described by the internal stress $\tau_{\text {int }}$ that can be computed from the plastic slip, either using a finite-element framework as in Lemarchand et al. [26] or, for an infinite body, using a Green's function as done in the present work.

It is clear that if the elementary volume is such as to encompass multiple dislocations, the interactions between these dislocations are in danger of becoming lost in an averaged description. Let us ask, what are the features of the dislocation system that we can no longer see if, as in the present model, our eyes are blurred to the extent that we lose vision of individual dislocations? We can still see the presence of dislocations (the variable $\rho$ ) as well as excess Burgers vector (the variable $\kappa$ ), but we can no longer see whether the excess dislocations are arranged in embryonic walls or in small pile-ups. Equally, for dislocations of zero net Burgers vector, we can no longer see whether they are arranged in loose multipoles or in narrow, isolated dipoles and what would be the height of these dipoles (the slip plane spacing of adjacent dislocations). Yet, this information matters; it is in fact essential for the dynamics: Dislocations, if arranged in dipoles, will not move at stresses below the dipole-breaking stress. Similarly, dislocations of the same sign forming an embryonic pile-up will jointly push against an obstacle, whereas dislocations of the same sign forming an embryonic wall will mutually reduce the forward stress on such an obstacle.

Since information is being lost if one coarse-grains on scales above the MDS, yet the physical reality to be described cannot depend on the level of resolution, it is mandatory that additional terms must emerge, which in our equations are represented by the additional stresses $\tau_{\mathrm{f}}, \tau_{\text {back }}$, and $\tau_{\text {diff }}$. Formally, these stress terms relate to dislocation-dislocation correlations which are bound to come into play if averages are computed in a mathematically correct manner; regarding this aspect we refer the reader to Groma et al. [12] and Valdenaire et al. [11]. Here, we focus on the physics.

$\tau_{\mathrm{f}}$ stems from the action, on a given dislocation, of dislocations of the opposite sign in its close vicinity. We may call it a dipole-like stress. The action of this stress depends on whether or not the stress acting on the dipole from outside is sufficient to break the dipole. If it is not (static case), the internal interaction of the dislocations in the dipole/multipole offsets the acting stress and the dislocations do not move. In the moving phase, above the dipole-breaking stress, the repeated formation-and-breaking of dipoles leads to dissipation. We also note that this effect is not dependent on the sign of the acting stress. Hence, the overall picture is very much that of a dry-friction-like behavior. The magnitude of the "friction stress" is controlled by an internal length scale, namely the spacing of the slip planes of the dislocations that typically constitute a dipole, or in other words the dipole height (multipoles add an additional layer of complexity but do not change the fundamental picture). In our model, this quantity enters through the nondimensional parameter $\alpha$ which can be understood as being proportional to the characteristic dipole height or, more generally, to the characteristic spacing of opposite dislocations in mutual-trapping configurations, measured in units of MDS.

The stress $\tau_{\text {back }}$ is associated with gradients in the excess dislocation density; it is computed from a combination of correlation functions that strongly weights the interactions of dislocations of the same sign on the same or nearby slip planes. Accordingly, we may consider it a pile-up stress: If the density of positive excess dislocations ahead of a given positive dislocation is larger than behind it (positive gradient of $\kappa$ ), the dislocation is pushed back; if the gradient of $\kappa$ is negative, it is pushed forward. The nondimensional parameter $D$ can be understood as the typical spacing of dislocations of the same sign in piled-up configurations, again measured in units of MDS.

Least easy to interpret is the stress contribution $\tau_{\text {diff }}$ which pushes dislocations of opposite signs in the same direction. This "diffusion stress", which is controlled by the parameter $A$, accounts for the fact that dipoles and multipoles have finite extension, such that dislocation density cannot localize down to arbitrary narrow scales.

In summary, all three factors $\alpha, D$, and $A$ are proportional to spacings of individual dislocations, measured in units of MDS, with $\alpha$ mainly characterizing the spacing of slip planes of adjacent dislocations, $D$ spacing of dislocations of the same sign in piled-up configurations, and $A$ the extension of dipoles and multipoles in the glide direction.

\section{Initial conditions, boundary conditions, loading protocol}

We simulate a system of size $L \times L$, implementing periodic boundary conditions in $x$ and $y$ for the stresses and in $x$ for the dislocation fluxes. For the stresses this means that the convolution integral in Eq. (7) is evaluated using the $L$-periodically continued Fourier transform of the kernel. As initial conditions we use $\rho^{ \pm}(\boldsymbol{r}, t)=\rho_{0} / 2+\epsilon \delta \rho^{ \pm}(\boldsymbol{r}, t)$ where $\epsilon \ll 1$ and we consider two types of perturbation $\delta \rho^{ \pm}$: (i) a Gaussian white noise of unit standard deviation and (2) a localized Gaussian "blob" of width $l=\rho_{0}^{-1 / 2}$ located at the center of the simulation cell. We consider two loading protocols: In a creeplike protocol we impose a constant external stress $\tau_{\text {ext }}>1$ above the flow stress of the homogeneous system and keep it fixed throughout the simulation, thus implementing creeplike testing conditions. Alternatively, in a strain-rate controlled protocol we monitor the strain rate which is locally given by Eq. (1). We then continually adjust the 
external stress to maintain the spatial average of the strain rate at a fixed, externally imposed value, $\left\langle\partial_{t} \gamma\right\rangle=\dot{\gamma}_{\text {ext }}$. The quasistatic case is implemented by letting the imposed strain rate go to zero (in practice: to a very small value).

\section{DISCRETE STOCHASTIC MODEL}

Our second model considers dislocation motion to be driven by the same stress contributions as introduced in Sec. IIB. However, the implementation of the dynamics differs radically.

We now consider a spatially and temporally discrete model where space is discretized onto an $L \times L$ lattice consisting of $N \times N$ square unit cells of size $d \times d$ with $d=L / N$. The simulation lattice is aligned with the $x$ and $y$ axes of a Cartesian coordinate system where $(x, y) \rightarrow(i=x / d, j=y / d)$. The discrete coordinate $i$ marks the slip direction and $j$ the direction of the slip plane normal. Periodic boundary conditions are assumed. Again we consider densities $\rho^{ \pm}$of positive and negative dislocations; however, dislocation densities are now assumed to be constant over each lattice cell and to be "quantized" in units $\rho_{d}$ which are integer fractions $\rho_{d}=\rho_{0} / M$ of the overall dislocation density $\rho_{0}$. A discrete density quantum $\rho_{d}$ of sign $s \in\{1,-1\}$ is henceforth denoted as a positive or negative "dislocatom." The dislocation state of lattice site $(i, j)$ is then characterized by the densities $\rho_{i j}^{+}=n_{i j}^{+} \rho_{d}$ and $\rho_{i j}^{-}=n_{i j}^{-} \rho_{d}$ or equivalently by the respective dislocatom numbers $n_{i j}^{ \pm}$. Again, we consider the overall densities of positive and negative dislocations and hence the total dislocatom numbers to be conserved.

We evolve the quantized dislocation densities on the lattice in discrete steps. Since all dependent and independent variables of the problem can be expressed in terms of integer numbers, we are dealing with a cellular automaton (CA) dynamics for which we now specify the evolution rules.

\section{A. Cellular automaton dynamics}

The motion of dislocations is described as discrete shuffling of dislocatoms between sites that are adjacent in the $i$ direction. The motion of positive and negative dislocatoms is controlled by driving forces that are proportional to the same effective stresses $\mathcal{T}^{ \pm}$that govern dislocation transport in the continuum model, with the only difference that these stresses (and also the plastic strains) are now defined on the boundaries between cells $(i, j)$ and $(i+1, j)$ that are adjacent in the slip direction. This implies that the lattice used for stress evaluation is shifted with respect to the lattice used for dislocation density evolution by $d / 2$ along the direction of slip. Without loss of generality, we denote as boundary $(i, j)$ the boundary between cells $(i, j)$ and $(i+1, j)$.

Dislocatoms move across boundaries subject to the following rules:

(1) Dislocatoms do not move across boundaries experiencing zero effective stress.

(2) Among all boundaries experiencing nonzero effective stress we determine, in each given time step, a critical boundary and dislocatom sign defined as the boundary and sign for which the effective stress has the largest absolute value:

$$
\left(i_{\mathrm{m}}, j_{\mathrm{m}}, s_{\mathrm{m}}\right):\left|\mathcal{T}_{i_{\mathrm{m}} j_{\mathrm{m}}}^{S_{\mathrm{m}}}\right|:=\max _{i, j, s}\left|\mathcal{T}_{i j}^{s}\right| .
$$

Across this critical boundary, we move one dislocatom of sign $s_{\mathrm{m}}$ in direction $s_{\mathrm{m}} \operatorname{sgn}\left(\mathcal{T}_{i_{\mathrm{m}} j_{\mathrm{m}}}^{s_{\mathrm{m}}}\right)$. In other words, positive dislocatoms move to the right from site $(i \rightarrow i+1)$ under a positive stress and to the left from $(i+1 \rightarrow i)$ under a negative effective stress, while negative dislocatoms show the opposite behavior. After a dislocatom has moved across a boundary, the dislocatom numbers on both sites are adjusted accordingly.

(3) Motion of a dislocatom across a boundary changes the strain associated with this boundary. If a dislocatom moves from a site under a positive effective stress, then the strain $\gamma_{i j}$ on the crossed boundary is increased by $\rho_{d} b d$. If the dislocatom moves under a negative effective stress, the strain is decreased by the same amount.

(4) After a dislocatom has moved we recalculate all stresses (for details see below) and determine the next critical boundary.

These rules implement a CA with extremal dynamics, corresponding to a physical situation where the velocity of dislocations increases with effective stress in a very abrupt manner (e.g., an exponential law with a large exponent or a very high power law), such that the dislocation with the highest stress moves much faster than all others.

\section{B. Calculation of stresses}

The effective driving stresses $\mathcal{T}_{i j}^{ \pm}$are calculated from the same equations as for the transport model, with some adjustments for the discrete nature of the model and for the inclusion of stochastic terms. The total and excess dislocation densities are evaluated as $\rho_{i j}=\rho_{d}\left(n_{i j}^{+}+n_{i j}^{-}\right)$and $\kappa_{i j}=\rho_{d}\left(n_{i j}^{+}-n_{i j}^{-}\right)$. The external stress is constant throughout the system. Internal stresses are evaluated according to Eq. (7) with the convolution replaced by the discrete lattice sum. Back stresses and diffusion stresses are evaluated from Eqs. (9) and (10) with the spatial derivatives replaced by the respective directional difference quotients. The friction stress associated with the boundary $(i, j)$ is evaluated as

$$
\tau_{\mathrm{f}, i j}^{ \pm}=\alpha \mu b \sqrt{\rho_{i j}+\rho_{i+1, j}}\left(1 \mp \frac{\kappa_{i j}+\kappa_{i+1, j}}{\rho_{i j}+\rho_{i+1, j}}\right) \xi_{i j},
$$

where $\xi_{i j}$ is a Gaussian-distributed random variable of average 1 and standard deviation $\sigma_{\tau}$. After a dislocatom move across some boundary, a new value of this variable is assigned to the boundary from the same distribution. This feature allows the model to account for stress fluctuations arising from the changes in local configurations of the discrete dislocations. Setting $\sigma_{\tau}=0$ makes Eq. (14) the direct discrete counterpart of (12).

\section{Initial conditions, boundary conditions, loading protocol}

We impose periodic boundary conditions as in the continuum model. Initial conditions are constructed by placing $N \times N \times M / 2$ positive and an equal number of negative dislocatoms randomly on the simulation lattice sites. We use two different types of loading protocol: (i) We impose a constant stress as in the continuum model. (ii) Alternatively, we increase, after an initial relaxation step, the stress precisely to the value needed to create one critical boundary. We trace the subsequent relaxation until no critical boundaries are left, and repeat. This algorithm corresponds to a quasistatic (infinitely 
slow) increase of the external stress and produces a stress-strain curve that approaches a horizontal asymptote corresponding to the macroscopic flow stress. Note that in the CA, performing constant-stress simulations does not change the patterns at all since the sequence of dislocatom moves due to the extremal dynamics is unchanged if one adds a spatially constant external stress of arbitrary magnitude.

\section{RESULTS}

\section{A. Linear stability analysis of transport equations}

A linear stability analysis (LSA) of the dislocation transport equations in Sec. II has been performed by Groma et al. [12]. Here we repeat that analysis for the generic dislocation velocity law given by Eq. (3). The results serve as reference for comparison with the numerical investigation of the fully nonlinear equations and with the results obtained from the discrete stochastic model.

One considers a spatially homogeneous reference state $\rho^{+}=\rho^{-}=\rho_{0} / 2$ under external stress $\tau_{\text {ext }}$ and investigates the time evolution of infinitesimal perturbations around this state in linear approximation. Without loss of generality, we assume that $\tau_{\text {ext }}$ is positive; results for negative shear stress are equivalent but for a change in the sign of $\kappa$. The effective driving stress in the reference state is given by $\mathcal{T}^{+}=\mathcal{T}^{-}=\mathcal{T}_{0}$. Two cases need to be distinguished: The jammed case, $\tau_{\text {ext }}<$ $\alpha \mu b \sqrt{\rho_{0}}$, whence $\mathcal{T}_{0}=0$ and the system trivially remains stationary in the reference state, and the flowing case, $\tau_{\text {ext }}>$ $\alpha \mu b \sqrt{\rho_{0}}$, where $\mathcal{T}_{0}=\tau_{\text {ext }}-\alpha \mu b \sqrt{\rho_{0}}>0$. This is the case which we investigate in the following. Because of the general scaling invariance properties of dislocation systems, results can be expressed in a generic form where all dislocation densities are measured in units of $C_{\rho}=\rho_{0}$, all lengths in units of $C_{l}=\rho_{0}^{-1 / 2}$, all stresses in units of $C_{\tau}=\mu b \sqrt{\rho}$, all strains in multiples of $C_{\gamma}=b \sqrt{\rho}$, and all times in units of $C_{t}=\left(M_{0} \mu b^{2} \rho_{0} \mathcal{T}_{0}^{m-1}\right)^{-1}$. In the following we use these units throughout.

We consider the evolution of fluctuations $\delta \kappa=\delta \rho^{+}-\delta \rho^{-}$, which characterizes the excess or geometrically necessary dislocation density, and $\delta \rho=\delta \rho^{+}+\delta \rho^{-}$, which characterizes inhomogeneous fluctuations of the total dislocation density. The corresponding evolution equations read in scaled form

$$
\begin{aligned}
& \partial_{t} \delta \rho=-\left[\frac{m}{2} \partial_{x}\left(\delta \tilde{\mathcal{T}}^{+}-\delta \tilde{\mathcal{T}}^{-}\right)+\tilde{\mathcal{T}}_{0} \partial_{x} \delta \kappa\right] \\
& \partial_{t} \delta \kappa=-\left[\frac{m}{2} \partial_{x}\left(\delta \tilde{\mathcal{T}}^{+}+\delta \tilde{\mathcal{T}}^{-}\right)+\tilde{\mathcal{T}}_{0} \partial_{x} \delta \rho\right]
\end{aligned}
$$

where $\tilde{\mathcal{T}}_{0}=\tau_{\text {ext }} / \mu b \sqrt{\rho_{0}}-\alpha$ and

$$
\begin{aligned}
& \delta \tilde{\mathcal{T}}^{+}=-\frac{\alpha}{2} \delta \rho-A \partial_{x} \delta \rho+\alpha \delta \kappa-D \partial_{x} \delta \kappa+\tau_{\text {int }}(\delta \kappa), \\
& \delta \tilde{\mathcal{T}}^{-}=-\frac{\alpha}{2} \delta \rho+A \partial_{x} \delta \rho-\alpha \delta \kappa-D \partial_{x} \delta \kappa+\tau_{\text {int }}(\delta \kappa),
\end{aligned}
$$

where the long-range internal stress $\tau_{\text {int }}$ follows from $\delta \kappa$ by convolution with the dislocation shear stress field.
In Fourier space we get the following evolution equation for the Fourier modes of the perturbations $\delta \rho$ and $\delta \kappa$ :

$$
\frac{1}{m} \partial_{t}\left[\begin{array}{l}
\delta \rho \\
\delta \kappa
\end{array}\right]=\left[\begin{array}{ll}
-A k_{x}^{2} & -\left(\frac{\tilde{\mathcal{T}}_{0}}{m}+\alpha\right) i k_{x} \\
-\left(\frac{\tilde{\mathcal{T}}_{0}}{m}-\frac{\alpha}{2}\right) i k_{x} & -D k_{x}^{2}-T(\boldsymbol{k})
\end{array}\right]\left[\begin{array}{l}
\delta \rho \\
\delta \kappa
\end{array}\right],
$$

where the factor $T$ derives from the Fourier transform of the dislocation shear stress field as

$$
T(\boldsymbol{k})=i k_{x} \tau_{x y}^{d}(\boldsymbol{k})=\frac{1}{\pi(1-v)} \frac{k_{x}^{2} k_{y}^{2}}{k^{4}} .
$$

The growth rates of fluctuations follow from the equation for the eigenvalues of the characteristic equation of this linear system as

$$
\begin{aligned}
& \frac{1}{m} \Lambda^{ \pm}(\boldsymbol{k}) \\
& =-\frac{\left[(A+D) k_{x}^{2}+T(\boldsymbol{k})\right]}{2} \\
& \quad \pm \frac{\sqrt{\left[(A+D) k_{x}^{2}+T(\boldsymbol{k})\right]^{2}-4 k_{x}^{2}\left\{A\left[D k_{x}^{2}+T(\boldsymbol{k})\right]-B\right\}}}{2}
\end{aligned}
$$

where

$$
B=\left(\alpha+\frac{\tilde{\mathcal{T}}_{0}}{m}\right)\left(\frac{\alpha}{2}-\frac{\tilde{\mathcal{T}}_{0}}{m}\right),
$$

which in the limits $\mathcal{T}_{0} \rightarrow 0$ (slow deformation) or $m \rightarrow \infty$ (rate-independent behavior) reduces to

$$
B=\frac{\alpha^{2}}{2}
$$

Positive eigenvalues, corresponding to growing perturbation modes, exist if $B>0$ which, in the two limits of slow deformation or rate-independent behavior, is always fulfilled provided the condition for plastic flow, $\tilde{\mathcal{T}}_{0}>0$, is met. In the general case we find an unstable band of external stress values $\alpha \leqslant \tilde{\tau}_{\text {ext }} \leqslant \alpha(m+2) / 2$ over which spatially heterogeneous perturbations of a homogeneous dislocation arrangement are able to grow. Within the unstable regime the unstable modes extend over a band of unstable wave vectors $\boldsymbol{k}$ fulfilling the equation

$$
A D k_{x}^{2}+A T(\boldsymbol{k})-B<0 .
$$

Perturbations of maximum amplification have the wave vector $\boldsymbol{k}^{\max }$ with $k_{y}^{\max }=0$ and

$$
k_{x}^{\max }=\rho_{0}^{1 / 2}\left[2 B \frac{-1+\sqrt{1+\frac{(A-D)^{2}}{4 A D}}}{(A-D)^{2}}\right]^{1 / 2} .
$$

Hence, we expect the emerging patterns to be dominated by heterogeneities in the $x$ rather than $y$ direction. The wavelength is determined by the external stress level (parameter $B$ ) as well as by the parameters $A$ and $D$ which characterize the range of dislocation correlations as shown in Fig. 3. Two important limiting cases are the limit of slow deformation, $\tilde{\mathcal{T}}_{0} \rightarrow 0$, and the limit of rate independence, $m \rightarrow \infty$. In both limits, $B \rightarrow \alpha^{2} / 2$ and hence the critical wave vector depends only on the nondimensional parameters $A$ and $D$ that characterize 

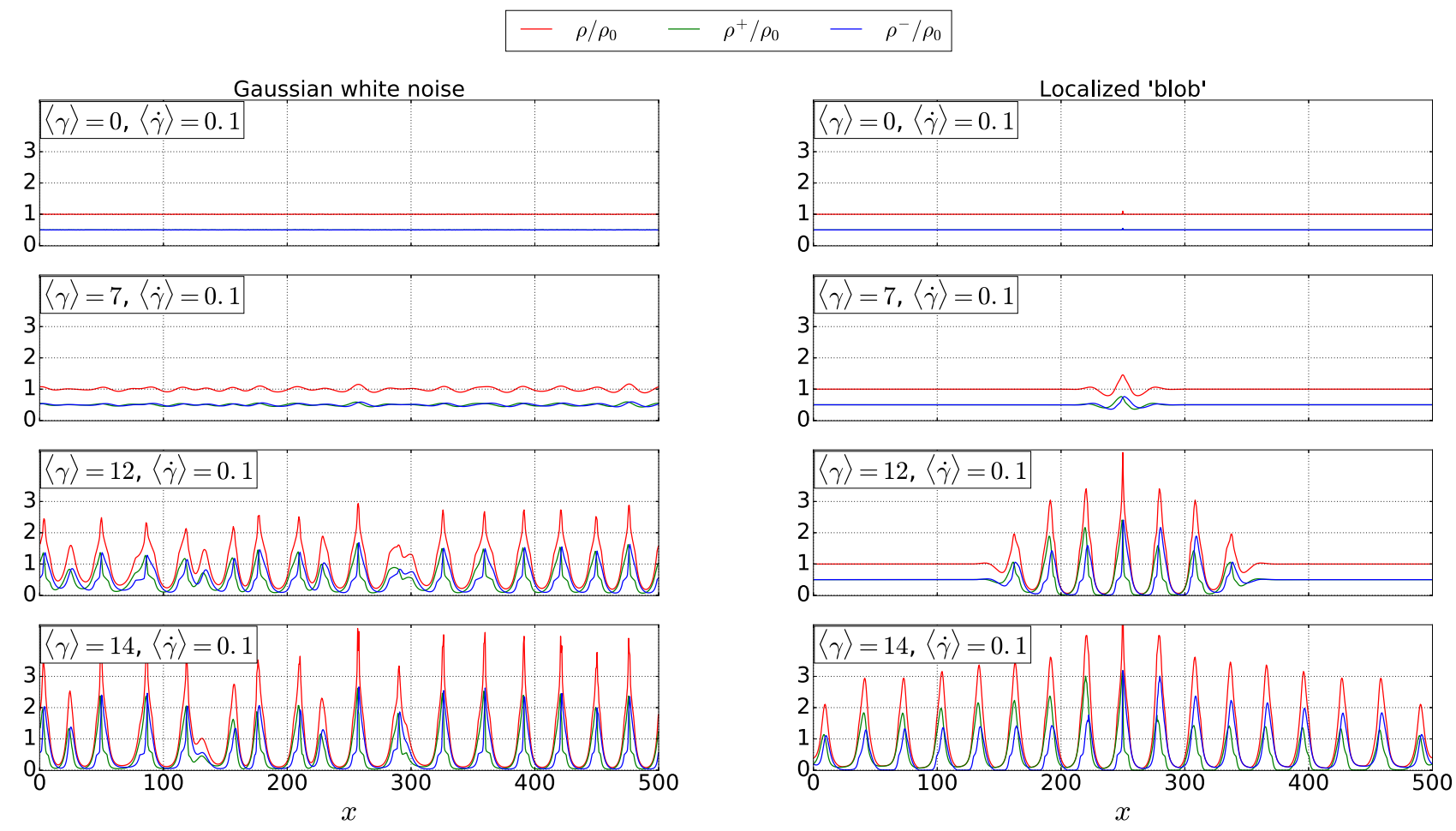

FIG. 1. Spatiotemporal evolution of dislocation density patterns for two different initial conditions; left: small Gaussian white noise superimposed on a homogeneous density distribution, right: localized density fluctuation superimposed on a homogeneous distribution; parameters $A=0.5, D=0.4, \alpha=0.3, \tau_{\mathrm{ext}}=1.1 \alpha$.

the range of dislocation correlations in units of dislocation spacings. This result implies that the dimensional pattern wavelength, $\lambda_{\max }=2 \pi / k_{\max }$, is proportional to the MDS in the reference state and inversely proportional to the corresponding flow stress, in line with the similitude principle.

\section{B. Simulations of the continuum transport equations \\ 1. Scenarios of pattern growth}

Given that LSA predicts the dominant unstable mode to be associated with heterogeneities along the $x$ but not the $y$ direction, we first investigate a one-dimensional scenario where we impose homogeneity in the $y$ direction, hence $k_{y}=0$ by construction. In the $x$ direction we use periodic boundary conditions with period $L=500$. We consider two types of initial conditions, namely (i) a Gaussian white noise and (ii) a small, localized dislocation density "blob" on top of the homogeneous background (Fig. 1). The amplitudes of the Fourier components of the perturbation are identical in both cases; however, in the case of the localized "blob" the phases are identical whereas for the white noise they are random. Assuming a white noise perturbation leads to spatially distributed growth of the patterns, whereas a localized blob as initial condition leads to a correlated growth scenario where a fully developed pattern emerges locally and then spreads through propagation of an enveloping wave. Despite the different growth dynamics, the fully developed patterns resulting from initial conditions (i) and (ii) are very similar in terms of morphology and wavelength.

Initially all Fourier modes of the perturbation have equal amplitude in both cases. The time evolution of the Fourier coefficients of the emergent patterns is shown in Fig. 2 (left) for case (i); case (ii) shows a practically identical behavior. From the initial growth rates of the discrete Fourier modes $\rho\left(k_{x}\right)$ we deduce growth factors defined as $\Lambda\left(k_{x}\right)=\Delta \ln \rho\left(k_{x}\right) / \Delta t$. Comparison with the analytical prediction of Eq. (19) shows excellent agreement as illustrated in Fig. 2 (right). The wavelengths $\lambda$ of the fully developed spatial patterns, determined as $\lambda=L / n_{\max }$ where $n_{\max }$ is the number of density maxima along the direction of the slip, match very closely (within 5\%) the predictions of linear stability analysis for the wavelength of the mode with maximum amplification. This observation, which holds throughout the parameter regime (Fig. 3), is remarkable since the nonlinearities have clearly a strong influence on the density distribution which is very different from a sinusoidal wave.
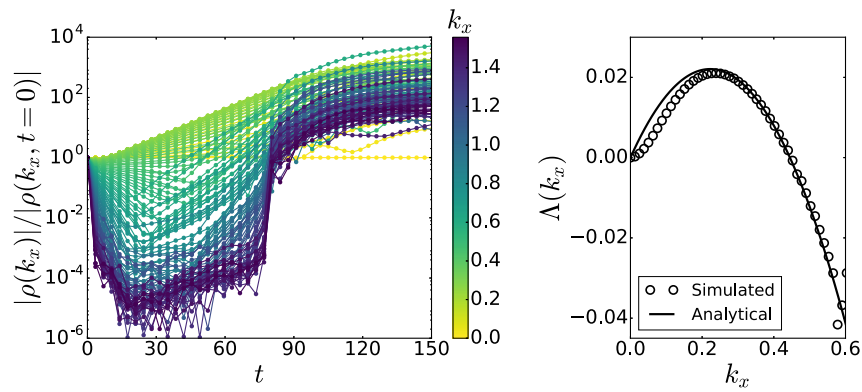

FIG. 2. Left: Evolution of the Fourier modes $\rho\left(k_{x}\right)$ of the total dislocation density $\rho(x)$; right: growth rates as deduced from the initial slope of the $\ln \left|\rho\left(k_{x}\right)\right|$ vs $t$ curves and analytical prediction according to Eq. (19); parameters as in Fig. 1. 

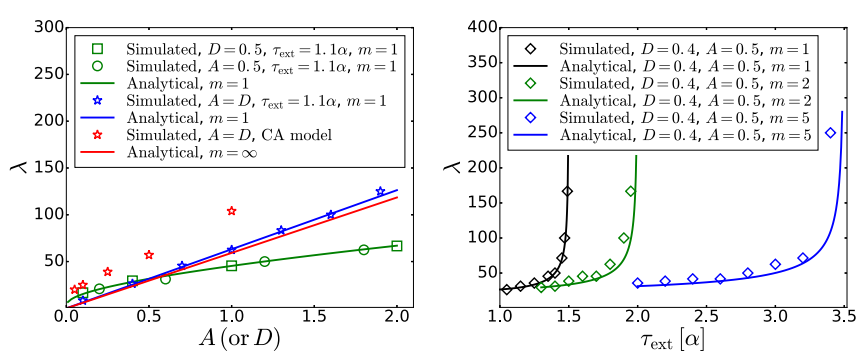

FIG. 3. Pattern wavelength $\lambda$ as function of the model parameters, left: $\lambda(A, D)$ values for $D=0.5$ (squares), $A=0.5$ (circles), and $A=D$ (stars), solid line: theoretical curve according to Eq. (23) (note that this expression is symmetrical with respect to an exchange of $A$ and $D), \tau_{\text {ext }}=1.1 \alpha$; right: $\lambda\left(\tau_{\text {ext }}, m\right)$ for $A=0.5$ and $D=0.4$ and different values of stress and stress exponent, solid lines: theoretical curves according to Eq. (23).

We then study the same patterning scenarios in two dimensions. In this case the emergent patterns have a stripelike character where the system is near-homogeneous in the $y$ direction whereas the $x$ dependency of the dislocation densities is almost identical to the one-dimensional case. If we use a Gaussian white noise as initial perturbation, embryonic patterns start growing locally and then, in a first "synchronization" stage, organize in the $y$ direction to form parallel walls. In a second "growth" stage the amplitude of these wall like dislocation density modulations increases while the once-established pattern remains in place (Fig. 4). If, on the other hand, we start from a localized dislocation density "blob" then an interesting scenario occurs (Fig. 5): The blob causes positive and negative dislocations to pile up from both sides. The long-range stresses of the double pile-up then lead to growth of a double wall similar to a kink band in the $y$ direction. Finally, the double wall serves as the nucleus for a nonlinear wave which spreads the pattern in the $y$ direction as in the one-dimensional case. Irrespective of the growth mode, the wavelength and morphology of the patterns are almost identical to the one-dimensional case.

Finally, Fig. 6 shows the Fourier spectrum of the emerging dislocation density distribution. We use a logarithmic scale; hence the color level can also be envisaged as an exponential growth factor, enabling direct comparison with Fig. 6, top left. It can be seen that the Fourier pattern of the developing pattern closely matches the growth predictions of linear stability analysis also in 2D (Fig. 6, top right). At later stages, nonlinear effects lead to growth also of initially damped shortwavelength modes (Fig. 6, bottom). This is in close analogy with the 1D observations shown in Fig. 2, left. Note that the growth of damped modes concerns mainly harmonics in $x$ of the initial unstable mode, as evidenced by the periodic striations of the Fourier patterns in Fig. 6, bottom.

\section{Structure of the fully developed patterns}

To elucidate the fully developed patterns, we refer for simplicity of illustration to the 1D case, as the two-dimensional patterns are homogeneous in the direction perpendicular to the slip plane. We define averaged stresses per dislocation as follows: the local flow stress is evaluated as the weighted average of the local "friction stresses," the local gradient dependent stress $\bar{\tau}_{\mathrm{g}}$ is evaluated as the weighted sum of back and diffusion stresses, and the local effective stress (the effective driving force per dislocation) is the sum of all stresses minus the flow stress. In nondimensional units we find

$$
\begin{aligned}
\bar{\tau}_{\mathrm{f}} & =\frac{\rho^{+} \tau_{\mathrm{f}}^{+}+\rho^{-} \tau_{\mathrm{f}}^{-}}{\rho}=\sqrt{\rho}\left(1-\frac{\kappa^{2}}{\rho^{2}}\right), \\
\bar{\tau}_{\mathrm{g}} & =\tau_{\text {back }}+\frac{\rho^{+} \tau_{\mathrm{diff}}^{+}+\rho^{-} \tau_{\mathrm{diff}}^{-}}{\rho}=-\frac{D}{\alpha \rho} \partial_{x} \kappa+\frac{A}{\rho} \frac{\kappa}{\rho} \partial_{x} \rho, \\
\bar{\tau}_{\text {eff }} & =\tau_{\text {ext }}+\tau_{\text {int }}+\bar{\tau}_{\mathrm{g}}-\bar{\tau}_{\mathrm{f}} .
\end{aligned}
$$

The fully developed patterns shown in Fig. 7 consist of periodic walls of high dislocation density separated by dislocationdepleted channels. The increased dislocation density in the walls increases the local flow stress $\tau_{\mathrm{f}}$; the opposite happens in the dislocation-depleted channels. This results in piling up of positive dislocations on the left and of negative dislocations on the right side of the walls. The resulting dislocation density gradient-dependent stresses (in our model: diffusion and back stresses) enhance the applied stress in the walls and reduce it in the channels until a balance is established. The internal stress $\tau_{\text {int }}$ is everywhere zero whereas the effective stress $\bar{\tau}_{\text {eff }}$ assumes a small positive value which, in load-controlled
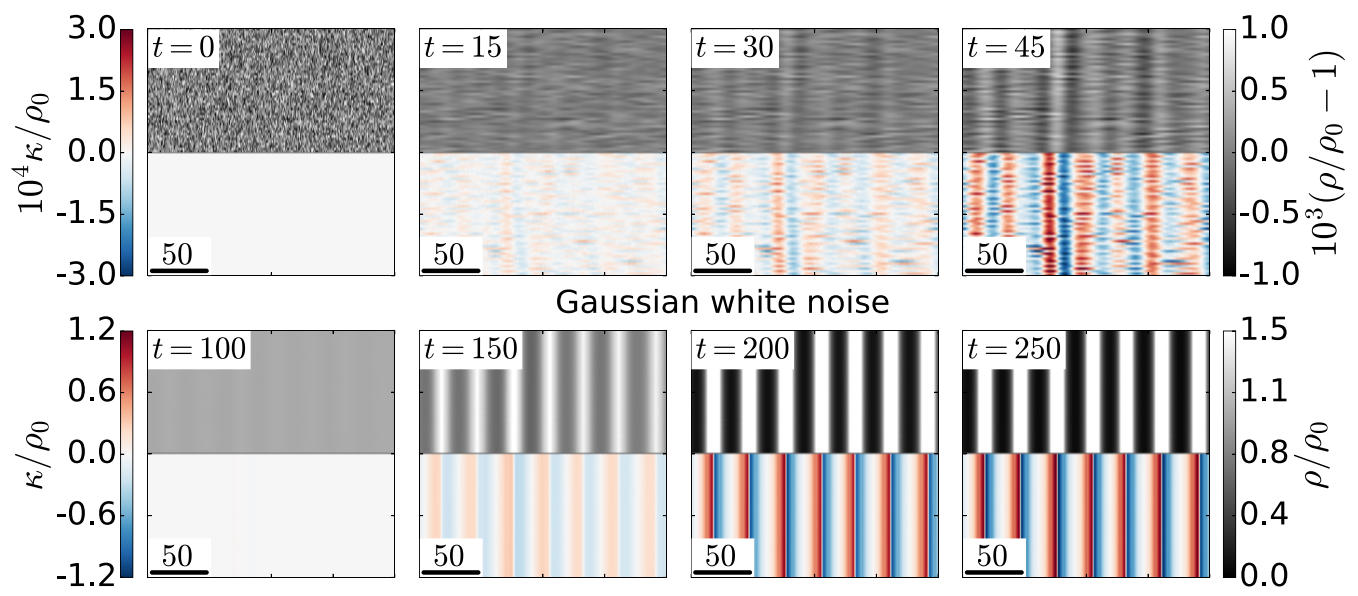

FIG. 4. Spatiotemporal evolution of dislocation density patterns [total density $\rho(\boldsymbol{r})$ and excess density $\kappa(\boldsymbol{r})$ ] in two dimensions for small Gaussian white noise superimposed on a homogeneous density distribution as initial condition; parameters as in Fig. 1. 

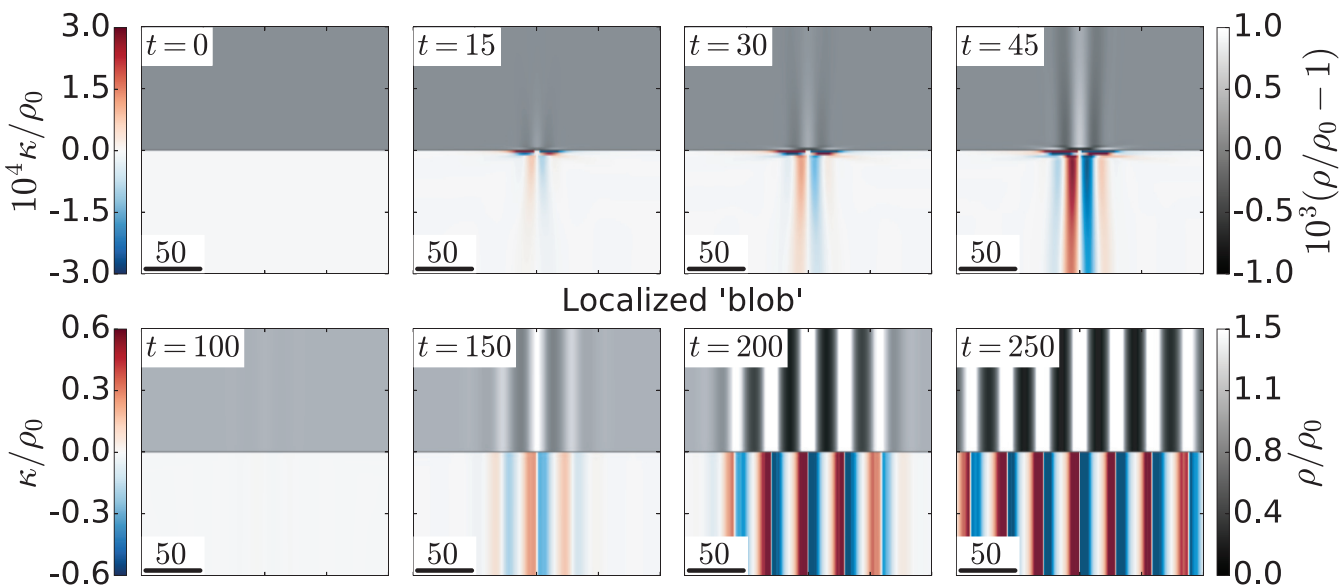

FIG. 5. Spatiotemporal evolution of dislocation density patterns [total density $\rho(\boldsymbol{r})$ and excess density $\kappa(\boldsymbol{r})$ ] in two dimensions for a localized dislocation density "blob" superimposed on a homogeneous density distribution as initial condition; parameters as in Fig. 1.

deformation, precisely corresponds to the stress level needed to maintain the imposed deformation rate. We note that the task of balancing the flow-stress heterogeneity, which is in the present model accomplished by the diffusion and back stresses, may alternatively be accomplished in a 3D model by line tension effects as considered in the model by Kratochvil of dislocation patterning in cyclic deformation [27]. We note that an equivalent role of line-tension and back-stress effects was also discussed in different contexts; see the study of Forest and Sedlacek on constrained plastic flow [28].

\section{Simulations of the stochastic cellular automaton model}

Simulations of the stochastic cellular automaton model were conducted using a quasistatic stress increase protocol as described in Sec. III C with the dislocatom size corresponding to $M=16$. The ensuing pattern formation is illustrated in Fig. 8. We can see the emergence of alternating walls of positive
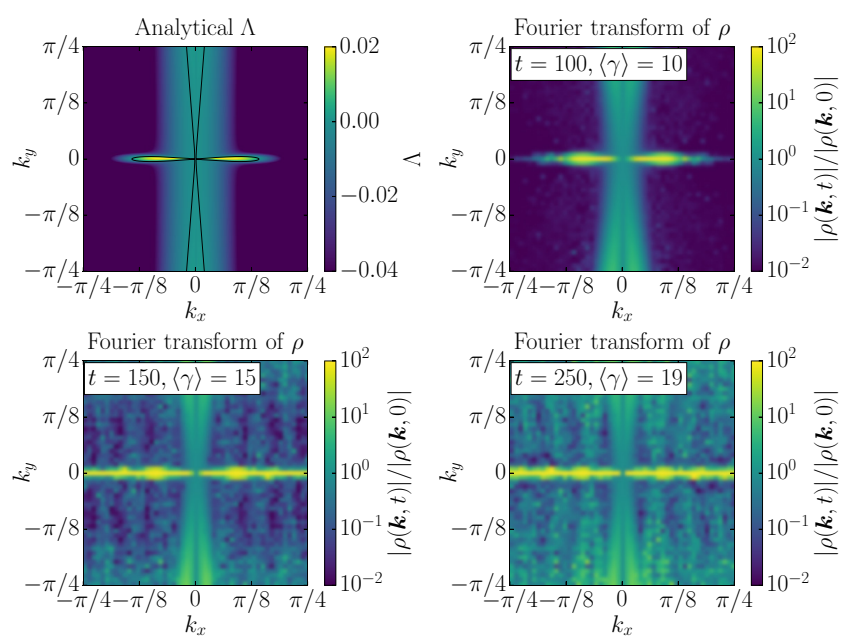

FIG. 6. Top left: Analytical growth factor $\Lambda$ as in Fig. 2; top right: normalized Fourier pattern $\rho(\boldsymbol{k}, t)$ for $t=100 C_{t}$, strain $\langle\gamma\rangle=10$; bottom: Fourier patterns $\rho(\boldsymbol{k}, t)$ for $t=150 C_{t}$, strain $\langle\gamma\rangle=15$ and $t=250 C_{t},\langle\gamma\rangle=19$; parameters as in Fig. 1 . and negative dislocations which become more pronounced with increasing strain.

The Fourier transform of the emergent patterns, taken at different strains, points to a growth scenario that differs substantially from that in the deterministic transport model. While the overall symmetry of the Fourier pattern matches the observations from the deterministic transport model and the corresponding linear stability analysis results, Fig. 9 demonstrates that the dominant wavelength of the patterns obtained from the CA shifts in the course of patterning from shorter to longer wavelengths (smaller $k_{x}$ ). This may be a feature of the short-wavelength noise that is inherent in the CA dynamics: The deterministic transport dynamics leads to a growth of the initially present spatial fluctuations that initially follows the LSA predictions. The CA dynamics, by contrast, continually adds spatiotemporal noise at the shortest possible
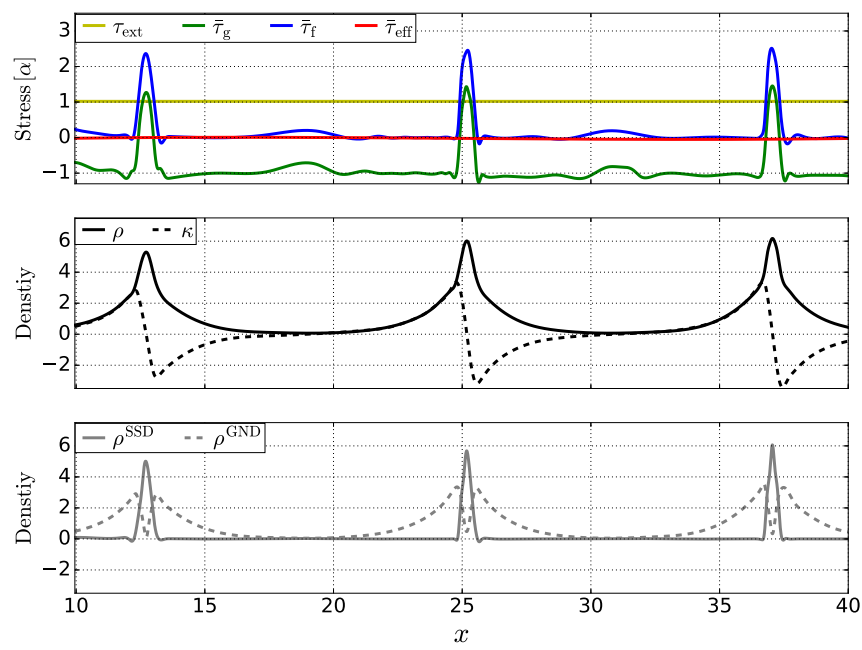

FIG. 7. Stresses and dislocation densities in a fully developed pattern; top: external stress, flow stress, and gradient-dependent stress as defined in Eq. (24); center: densities $\rho$ and $\kappa$ as used in our computation; bottom: "geometrically necessary" density $\rho_{\mathrm{GND}}=|\kappa|$ and "statistically stored" density $\rho_{\mathrm{SSD}}=\rho-\rho_{\mathrm{GND}}$; parameters: $\alpha=$ $0.3, A=D=0.2$, strain rate controlled deformation with $\dot{\gamma}_{\mathrm{ext}}=$ 0.01 . 


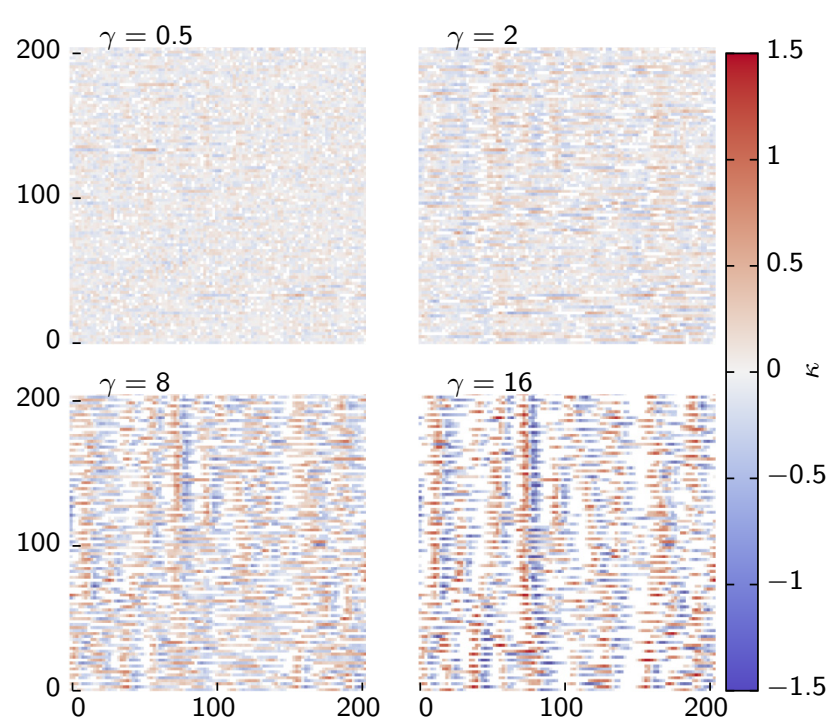

FIG. 8. Spatiotemporal evolution of dislocation density patterns [excess density $\kappa(\boldsymbol{r})$ ] as obtained from the stochastic CA model using a quasistatic loading protocol; parameters $A=D=0.1, \alpha=0.3$.

scale wavelength, namely on the scale of a single simulation cell. A second consequence of the "noisy" dynamics is that the final patterns are much less clearly developed than in the continuum model. While the Fourier transforms shown in Fig. 9 have been averaged over multiple realizations to eliminate the noise, this is unfortunately not possible for the spatial patterns since a superposition of multiple realizations would completely wash out the spatial features. If we reduce the noise by refining the $\mathrm{CA}$ mesh and reducing the dislocatom size accordingly, the results of the CA dynamics approach those of the continuum model.
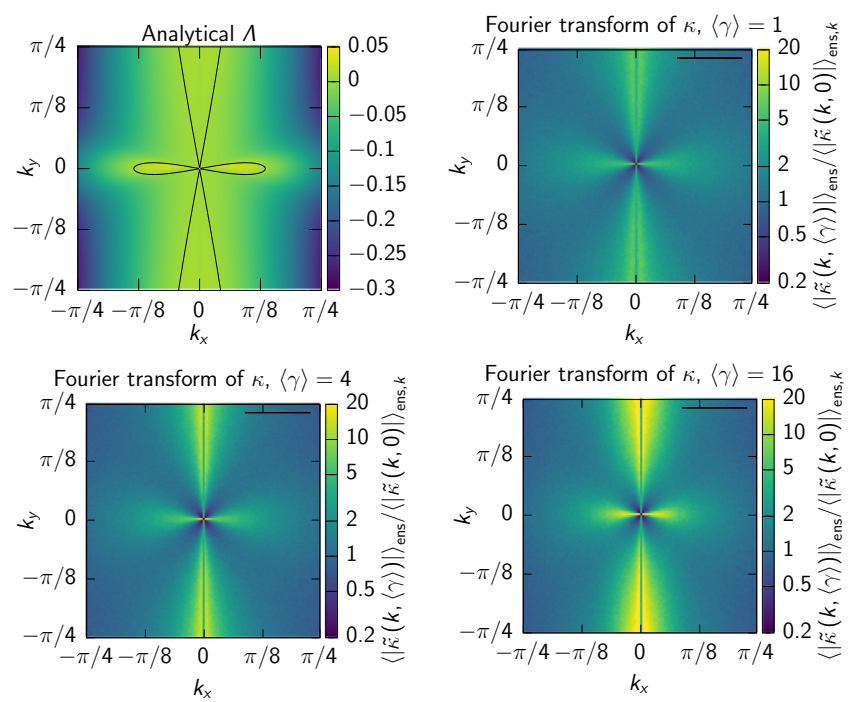

FIG. 9. Top left: Analytical growth factor $\Lambda$ according to Eq. (19) at $A=D=0.5$; top right: normalized Fourier patterns $\kappa(\boldsymbol{k}, \gamma)$ for mean strain $\langle\gamma\rangle=1$; bottom: Fourier patterns $\kappa(\boldsymbol{k}, \gamma)$ for mean strains $\langle\gamma\rangle=4$ and $\langle\gamma\rangle=16$, the Fourier patterns have been averaged over 100 realizations of the CA dynamics; cell size $d=2$.
In agreement with the LSA the wavelength of the fully developed patterns increases with increasing $A$ or $D$ as illustrated by the stars in Fig. 3. However, at larger $A$ or $D$ values the characteristic wavelengths obtained are larger than is predicted by the LDA. This can be attributed to the extremal dynamics used in the CA model. Moreover, the patterns obtained from the CA model are much more "noisy" than their deterministic counterparts. This is evident, for instance, from the absence of the higher-order "satellites" in the Fourier patterns of Fig. 9.

Repeating the simulations at constant external stress leads to results that are virtually identical with those derived from the quasistatic loading protocol. This is to be expected, since it is in the nature of the extremal dynamics that the addition of a constant stress, whatever its magnitude, does not change the sequence of events dictated by the extremal rule. As a consequence, the pattern wavelengths are stress independent: Whatever the stress level, the fully developed patterns match those obtained from the transport model in the limit $\tau_{\text {ext }} \rightarrow$ $\alpha \mu b \sqrt{\rho_{0}}$ which represents the case of deformation at vanishing rate. This limit actually represents the physically relevant case since, in real patterning scenarios, the rate-dependent contribution to the flow stress is exceedingly small. For illustration, we take typical parameters of $\mathrm{Cu}$ where $M_{0} \approx 2 \times 10^{4} \mathrm{~Pa}^{-1} \mathrm{~s}^{-1}$ (see Kubin and Canova [29]) and $b=2.54 \times 10^{-10} \mathrm{~m}$ and assume a dislocation density $\rho_{0}=10^{12} \mathrm{~m}^{-2}$. A typical strain rate of $10^{-3} \mathrm{~s}^{-1}$ then requires a stress on the order of $1 \mathrm{~Pa}$ which is about 7 orders of magnitude below the typical level of the dislocation interaction stresses; hence, the characteristic deviation of the applied stress from the value $\tau_{\mathrm{ext}}=\alpha \mu b \sqrt{\rho_{0}}$ is expected to be negligible.

\section{DISCUSSION AND CONCLUSIONS}

Nonlinear simulations of a simple model of dislocation density patterning show that the fully developed patterns closely match the predictions derived from a simple linear stability analysis. The patterns depend little on the dynamical rules governing dislocation motion: Two different dynamic models, one assuming stress dependent viscous dislocation motion and the other an extremely jerky cellular automaton evolution with extremal dynamics, produce qualitatively similar results. Also, simulations of the viscous model for different initial conditions show that the initial conditions, while having appreciable influence on the transient behavior, are practically immaterial to the fully developed pattern. The final patterns are essentially governed by a quasistatic balance of the different stress contributions entering the model; they depend on a stress balance which makes dislocations rest in metastable configurations, but not on the way the dislocations move between such configurations. This provides some hints as to why dislocation patterns are similar in pure and solutehardened fcc metals, or in fcc metals and ionic crystals with $\mathrm{KCl}$ structure, where the dislocation velocity laws are surely very different.

Looking at the balance of stresses involved we see three different kinds of stresses, which only in their mutual interplay can produce the observed patterning: First, we have an external stress driving the dislocation system. This is essential: no patterning can take place in the absence of plastic flow. 
Second, we have the stress contributions $\tau_{\text {int }}, \tau_{\text {back }}$, and $\tau_{\text {diff }}$ which derive from an energy functional comprising elastic and defect energy contributions. These stresses are essential for understanding the pattern morphology and wavelength; in particular, the wall-like morphology of the patterns stems from the structure of the elastic energy functional and the corresponding stress kernel governing $\tau_{\text {int }}$ : Minimization of $\tau_{\text {int }}$ forces the emergent walls to orient perpendicular to the slip plane. On the other hand the pattern wavelength depends on the parameters $A$ and $D$ which control the defect energy contribution to the energy functional that gives rise to gradient-dependent stress contributions. (Note also here the analogy with line tension effects that also are a direct consequence of defect energy - namely dislocation line energy — contributions). It is, however, important to note that the internal energy related stress contributions alone cannot explain pattern formation: In fact, the patterning process depends crucially on a fourth stress contribution which is dissipative in nature, namely the friction stress $\tau_{\mathrm{f}}$. Hence, the present patterning scenario cannot be envisaged as "energetically driven." In fact the basic mechanism leading to instability can be understood as a fundamental flux instability. To this end we envisage the nonsigned dislocation flux in a homogeneous dislocation system,

$$
\mathcal{J}_{\rho}=\rho v=M_{0} b \rho\left(\tau_{\text {ext }}-\alpha \mu b \sqrt{\rho}\right)^{m} .
$$

Taken as a function of $\rho$, this flux increases for small $\rho$, reaches a maximum, and then decreases towards zero when $\alpha \mu b \sqrt{\rho}=$ $\tau_{\text {ext }}$ and the dislocation system gets jammed. The location of the maximum is derived as

$$
\partial \mathcal{J}_{\rho} / \partial \rho=0, \quad \tau_{\text {ext }}-\frac{m+2}{2} \alpha \mu b \sqrt{\rho_{0}}=0 .
$$

Now we note that the instability condition $B<0$, Eq. (20), can be rewritten in dimensional variables as $\tau_{\text {ext }}-\frac{m+2}{2} \alpha \mu b \sqrt{\rho_{0}}<$ 0 . Instability thus occurs whenever the system operates on the descending branch of the $\mathcal{J}(\rho)$ curve: If an increase in dislocation density decreases the local dislocation flux, then more dislocations accumulate in such a location. This is the "dynamic" patterning scenario of Nabarro [6], however, with the twist that without accounting for the "energetic" stress contributions it is impossible to understand the pattern wavelength. We note that our observation-dislocation patterning occurs if the system operates on the descending branch of the $\mathcal{J}(\rho)$ curve-is equivalent to the observation reported by Zhu et al. [25] that, under conditions of strain rate control, dislocation patterning occurs in the regime where the flow stress is an increasing function of dislocation density.

We come thus to the conclusion that the past discussion about dislocation patterning may have been to some extent marred by false dichotomies and misleading analogies. Dislocation patterns are neither dynamic dissipative structures nor is their formation driven by energy minimization. Rather, the patterns emerge from the attempt of the dislocation system to minimize a coarse-grained energy functional while driven by an external stress and constantly encumbered by trapping into local, "microscopic" energy minima corresponding to jammed dislocation configurations [24,30]. On the coarse-grained scale such jamming leads to a friction-like stress that increases with increasing dislocation density, giving rise to a hydrodynamic type of dislocation transport instability. Past analogies, be they with spinodal decomposition or dynamic chemical waves, have in our opinion not been very helpful to understanding this interplay. Nevertheless the use of metaphors for conceptualizing dislocation patterns has a long (if somewhat murky) tradition and we cannot help coming up with a metaphor of our own: We think that the emergence of dislocation patterns from a hydrodynamic instability resembles the processes governing the emergence of traffic jams. The idea that a flux of vehicles that is a decreasing function of vehicle density is prone to instability has been long known in traffic theory. Here we find that the same instability scenario constitutes an intrinsic feature of dislocation motion.

Of course, the model discussed here does not capture all the features that are relevant to the morphology of real dislocation patterns. Rather, it should be considered as a maximally simplified model which strips the processes leading to dislocation patterning down to the bare essentials. Similarly to the Ising model in ferromagnetism that does not describe the actual behavior of any real ferromagnet but captures essential features of the ferromagnetic phase transition, we believe that the present model, while not describing the detailed morphology of any real dislocation pattern, captures the essential mechanism leading to dislocation patterning. To corroborate this conjecture we provide, in the Appendix, a discussion of some generalized models which account for screw dislocation motion, dislocation multiplication and annihilation, and dynamics of curved dislocations. We demonstrate that the inclusion of phenomenological terms describing dislocation multiplication and annihilation does not change the instability scenario: In the asymptotic limits of slow deformation or of rate-independent behavior, these terms become irrelevant for stability whereas in the general case, as we demonstrate for some models proposed in the literature, they lead to only very minor changes of the instability regime. We furthermore demonstrate that the present instability scenario carries over to models which describe motion of curved dislocations, and we take a look at such a model to show why screw dislocations do not form patterns. Further work is required to generalize the present analysis to multiple slip systems, and of course a large amount of effort is still needed to include all physically relevant details such as cross slip and climb processes, dislocation reactions in multislip deformation, surface and interface effects, lattice friction, and other factors that influence the precise morphology of dislocation patterns observed in a specific material and under specific deformation conditions.

\section{ACKNOWLEDGMENTS}

M.Z. and R.W. acknowledge financial support by DFG within the framework of the research unit FOR1650 "Dislocation based plasticity" under Grant No. Za171/7-1. M.Z. also acknowledges support by the Chinese State Administration of Foreign Experts Affairs under Grant No. MS2016XNJT044. I.G. and P.D.I. have been supported by the National Research, Development, and Innovation Foundation of Hungary (Projects No. NKFIH-K-119561 and No. NKFIH-KH-125380) and the Czech Science Foundation (P.D.I., Project No. 15-10821S). P.D.I. is also supported by the János Bolyai Scholarship of 
the Hungarian Academy of Sciences and by the ÚNKP-17-4 New National Excellence Program of the Ministry of Human Capacities.

\section{APPENDIX: GENERALIZATIONS}

\section{Dislocation multiplication and annihilation}

Our basic patterning scenario assumes conserved dislocation densities. In general, dislocations are created and annihilate, and both processes may affect patterning. In regions of enhanced dislocation density, dislocation flow is impeded and, as a consequence, dislocation multiplication may be inhibited. Furthermore, increased dislocation density may facilitate dislocation annihilation. It is thus plausible that both processes counteract the growth of dislocation-dense clusters and hence reduce the patterning tendency.

To assess this idea mathematically, we start out from the fundamental transport equations in the form of Eqs. (4) and add generation-annihilation terms $\tilde{\mathcal{R}}_{\rho}$ and $\tilde{\mathcal{R}}_{\kappa}$ to the right-hand side of these equations. To formulate these terms, we make the following generic observations:

(1) Because of Burgers vector conservation, neither the generation nor the annihilation of dislocations in the bulk can change the local net Burgers vector. Thus the generation or annihilation terms for excess (geometrically necessary) dislocations must vanish in the bulk, $\tilde{\mathcal{R}}_{\kappa}=0$.

(2) Because of symmetry between positive and negative dislocations, the generation term $\tilde{\mathcal{R}}_{\rho}(\rho, \kappa)$ can contain only even powers of $\kappa$. This implies that the lowest-order $\kappa$ dependent contribution is quadratic and thus irrelevant in the sense of linear stability analysis. For the purposes of linear stability analysis we are thus allowed to envisage $\tilde{\mathcal{R}}_{\rho}$ as a function of $\rho$ only.

(3) Both the multiplication and the annihilation of dislocations are consequences of their glide motion. We can therefore write the generation-annihilation dynamics as

$$
\tilde{\mathcal{R}}_{\rho}=\mathcal{J}_{\rho}(\rho) \Phi(\rho),
$$

where $\mathcal{J}_{\rho}=\rho v=\dot{\gamma} / b$ is the flux function introduced in the main paper and the function $\Phi(\rho)$ characterizes the generationannihilation kinetics.

With these observations and notations, the generalized transport equations become

$$
\begin{aligned}
& \partial_{t} \rho(\boldsymbol{r}, t)=-\partial_{x}\left(\rho^{+} v^{+}-\rho^{-} v^{-}\right)+\mathcal{J}_{\rho}(\rho) \Phi(\rho)+O\left(\kappa^{2}\right) \\
& \partial_{t} \kappa(\boldsymbol{r}, t)=-\partial_{x}\left(\rho^{-} v^{-}+\rho^{-} v^{-}\right)
\end{aligned}
$$

We now proceed to perform a linear stability analysis of these equations. In the presence of generation and annihilation, dislocation densities will in general reach a state of dynamic equilibrium of density $\rho_{0}$ such that $\Phi\left(\rho_{0}\right)=0$. The corresponding value of the dislocation velocity is $v_{0}$, and the flux function is $\mathcal{J}_{0}=\rho_{0} v_{0}$. We take this state as our reference state. The variation of the generation-annihilation terms around this state is then given by $\mathcal{J}_{0} \Phi^{\prime} \delta \rho$, where $\Phi^{\prime}=\partial \Phi /\left.\partial \rho\right|_{\rho_{0}}$.
Using the same scaling as in the main paper, the equations of evolution around the reference state read

$$
\begin{aligned}
& \partial_{t} \delta \rho=\left[\frac{m}{2} \partial_{x}\left(\delta \tilde{\mathcal{T}}^{+}-\delta \tilde{\mathcal{T}}^{-}\right)+\tilde{\mathcal{T}}_{0} \partial_{x} \delta \kappa\right]-\tilde{\mathcal{T}}_{0} \Psi^{\prime} \delta \rho, \\
& \partial_{t} \delta \kappa=\left[\frac{m}{2} \partial_{x}\left(\delta \tilde{\mathcal{T}}^{+}+\delta \tilde{\mathcal{T}}^{-}\right)+\tilde{\mathcal{T}}_{0} \partial_{x} \delta \rho\right],
\end{aligned}
$$

where

$$
\Psi^{\prime}=-\sqrt{\rho_{0}} \Phi^{\prime}
$$

The linear system changes to

$$
\partial_{t}\left[\begin{array}{l}
\delta \rho \\
\delta \kappa
\end{array}\right]=\left[\begin{array}{ll}
-m A k_{x}^{2}-\tilde{\mathcal{T}}_{0} \Psi^{\prime} & \left(m \alpha+\tilde{\mathcal{T}}_{0}\right) i k_{x} \\
\left(-\frac{m}{2} \alpha+\tilde{\mathcal{T}}_{0}\right) i k_{x} & -m D k_{x}^{2}-T(\boldsymbol{k})
\end{array}\right]\left[\begin{array}{l}
\delta \rho \\
\delta \kappa
\end{array}\right] .
$$

It is immediately evident that the LSA matrix is negatively definite in the short-wavelength limit $k_{\mathrm{x}} \rightarrow \infty$. The possibility of instability can thus be investigated by checking for zeros of the eigenvalues on the $k_{x}$ axis. Such zeros must fulfill the equation

$$
m^{2} A D k_{x}^{4}+m\left[A T(\boldsymbol{k})+D \tilde{\mathcal{T}}_{0} \Psi^{\prime}-B\right] k_{x}^{2}+T \tilde{\mathcal{T}}_{0} \Psi^{\prime}<0 .
$$

As $T=0$ on the $k_{x}$ axis, a sufficient condition for the existence of an unstable band of wave vectors is now given by $B-D \tilde{\mathcal{T}}_{0} \Psi^{\prime}>0$, which replaces the old condition $B>0$. The condition that the flux function $\mathcal{J}_{\rho}(\rho)$ must possess negative slope, Eq. (25), is thus replaced by the condition that this slope must exceed a critical steepness. This is remarkable because, in the limit of $\dot{\gamma} \rightarrow 0, \tilde{\mathcal{T}}_{0} \rightarrow 0$ whereas $B$ goes to a positive constant. Thus, if deformation is carried out at a sufficiently low rate, instability is bound to occur whatever the kinetics of multiplication and annihilation. The same is true in the rate-independent limit $m \rightarrow \infty$.

To investigate in quantitative terms the question to which extent the boundaries of the unstable regime are changed by introducing multiplication and annihilation under conditions away from these asymptotic limits, we need to specify a multiplication-annihilation model. We study two models, namely the well-known Kocks-Mecking model and a variant which considers a different dislocation density dependence of the multiplication term as proposed by Hähner and Zaiser [31]. Specifically,

$$
\begin{gathered}
\Phi_{\mathrm{KM}}=\beta \sqrt{\rho}-y_{\mathrm{a}} \rho, \quad \rho_{0, \mathrm{KM}}=\frac{\beta}{y_{\mathrm{a}}^{2}}, \\
\Phi_{\mathrm{HZ}}=\frac{\eta \tau_{\mathrm{ext}}}{\mu b}-y_{\mathrm{a}} \rho, \quad \rho_{0, \mathrm{HZ}}=\frac{\eta \tau_{\mathrm{ext}}}{\mu b y_{\mathrm{a}}} .
\end{gathered}
$$

Here, $\beta$ is a numerical parameter which can be understood as a multiplication distance in units of the dislocation spacing, $\eta$ characterizes the fraction of mechanical work that is stored in the form of dislocation line energy, and $y_{\mathrm{a}}$ is an annihilation cross section. With these relations we can write the parameters $\Psi^{\prime}$ as

$$
\begin{gathered}
\Psi_{\mathrm{KM}}^{\prime}=y_{\mathrm{a}} \sqrt{\rho_{0, \mathrm{KM}}}\left[1-\left(y_{\mathrm{a}} \sqrt{\rho_{0, \mathrm{KM}}}\right) / 2\right], \\
\Psi_{\mathrm{HZ}}^{\prime}=y_{\mathrm{a}} \sqrt{\rho_{0, \mathrm{HZ}}} .
\end{gathered}
$$


Experimental data indicate saturation dislocation densities of the order of $\rho_{0} \approx 10^{15} \mathrm{~m}^{-2}$ and annihilation distances of the order of $y_{\mathrm{a}}=1.6 \mathrm{~nm}$ for edge dislocations [32]. This leads to the estimate that $\Psi^{\prime} \leqslant 0.025$ for both models which implies that any corrections to the extension of the unstable regime are of the order of a few percent only. This consideration provides a quantitative justification of our decision to study patterning for the highly idealized conditions of a conserved dislocation system.

\section{Generalization to curved dislocations of general orientation}

A major limitation of the present investigation is that it considers straight and parallel dislocations only. For systems of curved dislocations, one may formulate transport equations which, similarly to those used in the present $2 \mathrm{D}$ models, allow for coexistence of dislocations of multiple orientations in the same volume element. The corresponding kinematic evolution equations have been developed under the label "continuum dislocation dynamics"; see Hochrainer et al. [8], Hochrainer [9], Monavari et al. [33], Monavari and Zaiser [34]. Here we present a generalization of the simplest CDD version by allowing, in analogy with the $2 \mathrm{D}$ dynamics discussed in the main paper, the velocity of dislocations to have both isotropic and orientation dependent contributions, such that the scalar velocity (the signed speed) of a dislocation with orientation $\boldsymbol{l}$ is given by $v(\boldsymbol{l})=v^{\mathrm{m}}+\boldsymbol{v}^{\mathrm{d}} \cdot \boldsymbol{l}=v^{\mathrm{m}}+v_{i}^{\mathrm{d}} l_{i}$. Note that while $v^{\mathrm{m}}$ is still the mean velocity averaged over all orientations, the directional velocity must now have a vectorial nature and the corresponding contribution changes its sign if we change the sign of the dislocation by replacing its line direction $\boldsymbol{l}$ with $-\boldsymbol{l}$. The asymmetry of the velocity is most pronounced for dislocations parallel to the directional vector $\boldsymbol{v}^{\mathrm{d}}$. We consider slip on a single slip system with the glide plane being the $x z$ plane of a Cartesian coordinate system and the Burgers vector pointing in $x$ direction. Curved dislocations may have any direction within the glide plane and, accordingly, the excess dislocation density is now described by a vector $\rho^{(1)}$ which points in the direction of the net dislocation orientation. This vector is perpendicular to the gradient of slip, $\rho^{(1)}=$ $(1 / b) \boldsymbol{\varepsilon} \cdot \nabla \gamma=(1 / b) \varepsilon_{i j} \partial_{j} \gamma$, where $\boldsymbol{\varepsilon}$ is the 2D Levi-Civita tensor in the glide plane, that rotates a vector within the glide plane counterclockwise by $90^{\circ}$. The total dislocation density is still described by a scalar $\rho$ which characterizes the line length per unit volume irrespective of orientation. Because the motion of curved dislocations perpendicular to their line direction changes their lengths, an additional curvature variable $q$ is required in CDD. The so-called curvature density $q$ gives the average angular content per line length per volume. For similarly oriented closed loops the integral of $q$ yields the number of loops. The named density variables, $\rho, \boldsymbol{\rho}^{(1)}$, and $q$, are the lowest-order terms of a list of higher-order fully symmetric alignment tensors $\boldsymbol{\rho}^{(2)}, \boldsymbol{\rho}^{(3)}, \ldots$ and $\boldsymbol{q}^{(1)}, \boldsymbol{q}^{(2)}, \ldots$

For a given speed $v(l)$ the evolution equations for $\rho, \boldsymbol{\rho}^{(1)}$, and $q$ may be obtained from Eqs. (217)-(219) of Hochrainer [9]. For the evolution of $q$ this requires consideration of the so-called rotation speed $\vartheta$ defined on a higherdimensional configuration space. The rotation speed of a curve segment is determined from a derivative of the speed along the curve. In this, the directional dependence of the velocity $v(\boldsymbol{l})$ introduces besides spatial derivatives a curvature contribution to $\vartheta$. Eventually, the evolution equations for the lowest-order CDD variables are obtained as

$$
\begin{aligned}
\partial_{t} \rho & =\partial_{i}\left(v^{\mathrm{m}} \varepsilon_{i j} \rho_{j}+v_{k}^{\mathrm{d}} \varepsilon_{i j} \rho_{k j}\right)+v^{\mathrm{m}} q-v_{j}^{\mathrm{d}} \varepsilon_{j k} q_{k}, \\
\partial_{t} \rho_{i} & =-\varepsilon_{i j} \partial_{j}\left(v^{\mathrm{m}} \rho+v_{k}^{\mathrm{d}} \rho_{k}\right), \\
\partial_{t} q & =-\partial_{i}\left(-v^{\mathrm{m}} q_{i}-v_{j}^{\mathrm{d}} q \varepsilon_{i j}+\partial_{j} v^{\mathrm{m}} \rho_{j i}+\partial_{j} v_{k}^{\mathrm{d}} \rho_{j k i}\right) .
\end{aligned}
$$

In these evolution equations appear the components $q_{i}, \rho_{i j}$, and $\rho_{i j k}$ of further alignment tensors. In order to obtain a closed system of equations, the higher-order terms may be expressed in terms of the given variables by the use of closure assumptions (cf. Monavari et al. [33], Hochrainer [9]).

In the sense of a linear stability analysis we now regard the evolution of small perturbations of an initially homogeneous and isotropic dislocation arrangement. For such weakly anisotropic arrangements we can use the following relations (see Hochrainer [9] and Monavari et al. [33]):

$$
\begin{aligned}
q_{i} & =\partial_{i} \rho_{i j}, \\
\rho_{i j} & =\frac{1}{2} \rho \delta_{i j}+O\left(\rho_{i}^{2}\right), \\
\rho_{i j k} & =O\left(\rho_{i}^{3}\right) .
\end{aligned}
$$

To make the analysis more transparent, we use a simplification which, however, does not reduce the generality of our stability considerations. Namely, we consider plane-wave-like perturbations where the projection of the wave vector on the $x z$ plane has the direction $\boldsymbol{e}_{s} \perp \boldsymbol{e}_{y}$. Derivatives in this direction are denoted as $\partial_{s}=\boldsymbol{e}_{s} \cdot \nabla$, and the corresponding projected wave vector is $\boldsymbol{k}_{s}=\boldsymbol{e}_{s}\left(\boldsymbol{k} \cdot \boldsymbol{e}_{s}\right)$. It then follows that the vector $\boldsymbol{\rho}$ can be written as $\kappa \boldsymbol{\varepsilon} \cdot \boldsymbol{e}_{s}$ where $\kappa$ is the scalar GND density. Nonzero $\kappa$ breaks the initial statistical isotropy of the system and defines a reference direction; hence, the directional velocity must have the structure $\boldsymbol{v}^{d}=v^{\mathrm{d}} \boldsymbol{\varepsilon} \cdot \boldsymbol{e}_{s}$. With these notations and neglecting terms of quadratic or higher order in the perturbations $\kappa, v^{\mathrm{d}}$, the evolution equations take the form

$$
\begin{aligned}
& \partial_{t} \rho=-\partial_{s}\left(v^{\mathrm{m}} \kappa+\frac{1}{2} v^{d} \rho\right)+v^{\mathrm{m}} q+v^{\mathrm{d}} \partial_{s} \rho, \\
& \partial_{t} \kappa=-\partial_{s}\left(\rho v^{\mathrm{m}}+\kappa v^{d}\right), \\
& \partial_{t} q=-\frac{1}{2}\left[\partial_{s}\left(v^{\mathrm{m}} \partial_{s} \rho+v^{d} q-\rho \partial_{s} v^{\mathrm{m}}\right] .\right.
\end{aligned}
$$

It is instructive to compare these equations with those for a $2 \mathrm{D}$ system. The structure of the transport terms is in fact very similar, with exception of a factor $(1 / 2)$ in the equation for $\rho$ that stems from the fact that only half of the total dislocation density is transported in the direction of $\boldsymbol{e}_{s}$ (but all of the excess density). However, additional generation terms appear in the balance of $\rho$ which relate to dislocation curvature and reflect the fact that motion of curved dislocations generally changes the line length per unit volume. Accordingly, an equation for curvature density emerges, as the motion of dislocations in an inhomogeneous velocity field may change their curvature.

We now need to define a reference state for stability analysis. A homogeneous reference state is given by $\rho=\rho_{0}, \kappa=0$, $q=q_{0}$ with the velocities $v_{0}^{\mathrm{m}}=v_{0}, v_{0}^{\mathrm{d}}=0$. This state is no longer stationary as expansion of curved loops continually increases the reference density which evolves according to $\partial_{t} \rho_{0}=q_{0} v_{0}$. This poses a conceptual problem, since perturbations which grow less rapidly than the reference density can hardly be taken to be indicators of patterning. The problem 
can however be resolved by scaling all variables as in the main paper and accounting for the time dependence of the scaling factor $\rho_{0}$. Hence we set $\tilde{\rho}=\rho / \rho_{0}, \tilde{\kappa}=\kappa / \rho_{0}$. Furthermore, if we scale lengths in units of $\rho^{-1 / 2}$ this imposes for $q$ the scaling $\tilde{q}=q / \rho^{3 / 2}$. The scaled variables fulfill in the spatially homogeneous case the equations

$$
\begin{aligned}
& \partial_{t} \tilde{\rho}=\tilde{v}_{0} \tilde{q}_{0}-\tilde{q}_{0} \tilde{v}_{0} \tilde{\rho}, \\
& \partial_{t} \tilde{\kappa}=-\tilde{q}_{0} v_{0} \tilde{\kappa}, \\
& \partial_{t} \tilde{q}=-\frac{3 \tilde{q}_{0} v_{0}}{2} \tilde{q} .
\end{aligned}
$$

It can be seen that the time-dependent scaling introduces terms which formally resemble a generation-annihilation dynamics for $\rho$. The stationary solution of the scaled homogeneous equations is $\tilde{\rho}=1, \tilde{\kappa}=\tilde{q}=0$. The evolution of perturbations around this state is in linear approximation, in scaled variables and dropping the tildes, given by

$$
\begin{aligned}
& \partial_{t} \delta \rho=-\partial_{s}\left(v_{0} \delta \kappa+\frac{1}{2} \delta v^{\mathrm{d}}\right)+v_{0} \delta q, \\
& \partial_{t} \delta \kappa=-\partial_{s}\left(v_{0} \delta \rho+\delta v^{\mathrm{m}}\right), \\
& \partial_{t} \delta q=-\frac{1}{2}\left(v_{0} \partial_{s}^{2} \delta \rho-\partial_{s}^{2} \delta v^{\mathrm{m}}\right),
\end{aligned}
$$

where we assume that $v_{0}$ depends on stress as in the main paper: $v_{0}=\tilde{\mathcal{T}}_{0}{ }^{m}$. To facilitate comparison, we also consider the additional stress contributions in the same form as in the main paper by setting

$$
\begin{aligned}
\delta v^{\mathrm{m}} & =m\left[-\frac{\alpha}{2} \delta \rho+\tau_{\text {int }}(\delta \kappa)-D \partial_{s} \delta \kappa\right], \\
\delta v^{\mathrm{d}} & =m\left(-A \partial_{s} \delta \rho+\alpha \delta \kappa\right) .
\end{aligned}
$$

The evolution of perturbations is then given, in Fourier space, by the matrix equation

$$
\frac{\partial_{t}}{m}\left[\begin{array}{l}
\delta \rho \\
\delta \kappa \\
\delta q
\end{array}\right]=\left[\begin{array}{lll}
-\frac{A}{2} k_{s}^{2} & -\left(\frac{\tilde{\mathcal{T}}_{0}}{m}+\frac{\alpha}{2}\right) i k_{s} & \frac{\tilde{\mathcal{T}}_{0}}{m} \\
-\left(\frac{\tilde{\mathcal{T}}_{0}}{m}-\frac{\alpha}{2}\right) i k_{s} & -D k_{s}^{2}-T(\boldsymbol{k}) & 0 \\
\frac{1}{2}\left(\frac{\tilde{\mathcal{T}}_{0}}{m}+\frac{\alpha}{2}\right) k_{s}^{2} & \frac{i k_{s}}{2}\left[T(\boldsymbol{k})+D k_{s}^{2}\right] & 0
\end{array}\right]\left[\begin{array}{l}
\delta \rho \\
\delta \kappa \\
\delta q
\end{array}\right],
$$

where the factor $T$ is now evaluated by superimposing the Fourier transforms of the shear stress fields of screw and edge dislocations according to

$$
T(\boldsymbol{k})=f_{\mathrm{e}} \frac{1}{\pi(1-v)} \frac{k_{x}^{2} k_{y}^{2}}{k^{4}}+f_{\mathrm{s}} \frac{1}{2 \pi} \frac{k_{z}^{2}}{k^{2}},
$$

with the notations $f_{\mathrm{e}}=k_{x}^{2} / k_{s}^{2}$ and $f_{\mathrm{s}}=k_{z}^{2} / k_{s}^{2}, k_{s}^{2}=k_{x}^{2}+k_{z}^{2}$, and $k^{2}=k_{x}^{2}+k_{y}^{2}+k_{z}^{2}$.

Looking at the system of equations which govern linear stability, we first consider the limit of quasistatic or rateindependent deformation when $\tilde{\mathcal{T}}_{0} / m \rightarrow 0$. In this limit, the equation for $\delta q$ decouples from the equations for $\delta \rho$ and $\delta \kappa$ and the system is then almost equivalent to the $2 \mathrm{D}$ system studied previously. In this case, the existence and extension of an unstable band of positive eigenvalues follows from a condition identical to Eq. (22) with the only difference that $B$ is now replaced by the slightly modified expression

$$
B=\left(\alpha+\frac{2 \tilde{\mathcal{T}}_{0}}{m}\right)\left(\frac{\alpha}{2}-\frac{\tilde{\mathcal{T}}_{0}}{m}\right),
$$

which in the athermal rate independent limit yields the same result as for the $2 \mathrm{D}$ case, $B=\alpha^{2} / 4$. Differences with respect to the $2 \mathrm{D}$ case of straight parallel edge dislocations arise, however, if we take the wave vector of the perturbation in a direction other than the $x$ direction. For instance, we may consider perturbations in the $z$ direction, corresponding to patterning of screw dislocations, such that $\boldsymbol{k}=k \boldsymbol{e}_{z}$. In this case, $T(\boldsymbol{k})=1 /(2 \pi)$ and Eq. (22) may be difficult to fulfill because the numerical value of $B$ cannot exceed the value $\alpha^{2} / 2 \approx 0.05$. To match the instability condition, we would need to require $A \leqslant \pi \alpha^{2} \approx 0.1$; however, then the critical wavelength becomes comparable to the MDS and the description of patterning in terms of dislocation density variations becomes spurious. Hence, we conclude that, under single slip conditions, pattern-forming instability of an arrangement of screw dislocations is suppressed because such patterns would induce long-range internal stresses which are absent or at least significantly reduced in a structure of edge dislocation walls. This is in line with the well-known observation that systems of long parallel screw dislocations, which dominate plasticity in low-temperature deformation of bcc metals, are homogeneously distributed and do not show appreciable patterning.
[1] N. Hansen and D. Kuhlmann-Wilsdorf, Low-energy dislocation-structures due to unidirectional deformation at low-temperatures, Mater. Sci. Eng. 81, 141 (1986).

[2] D. L. Holt, Dislocation cell formation in metals, J. Appl. Phys 41, 3197 (1970).

[3] D Walgraef and E. C. Aifantis, Dislocation patterning in fatigued metals as a result of dynamical instabilities, J. Appl. Phys. 58, 688 (1985).

[4] J. Pontes, D. Walgraef, and E. C. Aifantis, On dislocation patterning: Multiple slip effects in the rate equation approach, Int. J. Plasticity 22, 1486 (2006).

[5] J. M. Salazar, R. Fournet, and N. Banal, Dislocation patterns from reaction-diffusion models, Acta Metall. Mater. 43, 1127 (1995).
[6] F. R. N. Nabarro, Complementary models of dislocation patterning, Philos. Mag. A 80, 759 (2000).

[7] T. Hochrainer, M. Zaiser, and P. Gumbsch, A three-dimensional continuum theory of dislocation systems: Kinematics and meanfield formulation, Philos. Mag. 87, 1261 (2007).

[8] T. Hochrainer, S. Sandfeld, M. Zaiser, and P. Gumbsch, Continuum dislocation dynamics: Towards a physical theory of crystal plasticity, J. Mech. Phys. Solids 63, 167 (2014).

[9] T. Hochrainer, Multipole expansion of continuum dislocations dynamics in terms of alignment tensors, Philos. Mag. 95, 1321 (2015).

[10] I. Groma, F. F. Csikor, and M. Zaiser, Spatial correlations and higher-order gradient terms in a continuum description of dislocation dynamics, Acta Mater. 51, 1271 (2003). 
[11] P. L. Valdenaire, Y. Le Bouar, B. Appolaire, and A. Finel, Density-based crystal plasticity: From the discrete to the continuum, Phys. Rev. B 93, 214111 (2016).

[12] I. Groma, M. Zaiser, and P. D. Ispanovity, Dislocation patterning in a two-dimensional continuum theory of dislocations, Phys. Rev. B 93, 214110 (2016).

[13] I. Groma, G. Gyorgyi, and B. Kocsis, Dynamics of coarse grained dislocation densities from an effective free energy, Philos. Mag. 87, 1185 (2007).

[14] I. Groma, Z. Vandrus, and P. D. Ispanovity, Scale-Free Phase Field Theory of Dislocations, Phys. Rev. Lett. 114, 015503 (2015).

[15] M. Zaiser, Local density approximation for the energy functional of three-dimensional dislocation systems, Phys. Rev. B 92, 174120 (2015).

[16] T. Hochrainer, Thermodynamically consistent continuum dislocation dynamics, J. Mech. Phys. Solids 88, 12 (2016).

[17] R. Madec, B. Devincre, and L. P. Kubin, Simulation of dislocation patterns in multislip, Scr. Mater. 47, 689 (2002).

[18] C. Depres, C. F. Robertson, and M. C. Fivel, Low-strain fatigue in AISI 316L steel surface grains: A three-dimensional discrete dislocation dynamics modeling of the early cycles. I. Dislocation microstructures and mechanical behavior, Philos. Mag. 84, 2257 (2004).

[19] A. M. Hussein, S. I. Rao, M. D. Uchic, D. M. Dimiduk, and J. A. El-Awady, Microstructurally based cross-slip mechanisms and their effects on dislocation microstructure evolution in fcc crystals, Acta Mater. 85, 180 (2015).

[20] S. Sandfeld and M. Zaiser, Pattern formation in a minimal model of continuum dislocation plasticity, Modell. Simul. Mater. Sci. Eng. 23, 065005 (2015).

[21] S. Xia and A. El-Azab, Computational modeling of mesoscale dislocation patterning and plastic deformation of single crystals, Modell. Simul. Mater. Sci. Eng. 23, 055009 (2015).

[22] M. Sauzay and L. P. Kubin, Scaling laws for dislocation microstructures in monotonic and cyclic deformation of fcc metals, Prog. Mater. Sci. 56, 725 (2011).
[23] M. Zaiser and S. Sandfeld, Scaling properties of dislocation simulations in the similitude regime, Modell. Simul. Mater. Sci. Eng. 22, 065012 (2014).

[24] M.-C. Miguel, A. Vespignani, M. Zaiser, and S. Zapperi, Dislocation Jamming and Andrade Creep, Phys. Rev. Lett. 89, 165501 (2002).

[25] Y. Zhu, X. Niu, and Y. Xiang, Continuum dynamics of the formation, migration, and dissociation of self-locked dislocation structures on parallel slip planes, J. Mech. Phys. Solids 96, 369 (2016).

[26] C. Lemarchand, B. Devincre, and L. P. Kubin, Homogenization method for a discrete-continuum simulation of dislocation dynamics, J. Mech. Phys. Solids 49, 1969 (2001).

[27] J. Kratochvíl and R. Sedláček, Pattern formation in the framework of the continuum theory of dislocations, Phys. Rev. B 67, 094105 (2003).

[28] S. Forest and R. Sedlacek, Plastic slip distribution in two-phase laminate microstructures: Dislocation-based versus generalizedcontinuum approaches, Philos. Mag. 83, 245 (2003).

[29] L. P. Kubin and G. Canova, The modeling of dislocation patterns, Scr. Metall. 27, 957 (1992).

[30] G. Tsekenis, N. Goldenfeld, and K. A. Dahmen, Dislocations Jam at Any Density, Phys. Rev. Lett. 106, 105501 (2011).

[31] P. Hähner and M. Zaiser, Dislocation dynamics and work hardening of fractal dislocation cell structures, Mater. Sci. Eng. A 272, 443 (1999).

[32] U. Essmann and H. Mughrabi, Annihilation of dislocations during tensile and cyclic deformation and limits of dislocation densities, Philos. Mag. A 40, 731 (1979).

[33] M. Monavari, S. Sandfeld, and M. Zaiser, Continuum representation of systems of dislocation lines: A general method for deriving closed-form evolution equations, J. Mech. Phys. Solids 95, 575 (2016).

[34] M. Monavari and M. Zaiser, Annihilation and sources in continuum dislocation dynamics, Mater. Theory 2, 3 (2018). 